\title{
Probing new physics with long-lived charged particles produced by atmospheric and astrophysical neutrinos
}

\author{
Shin'ichiro Ando ${ }^{1}$, John F Beacom ${ }^{2,3,4}$, Stefano Profumo ${ }^{1,5}$ \\ and David Rainwater ${ }^{6}$ \\ ${ }^{1}$ California Institute of Technology, Pasadena, CA 91125, USA \\ 2 Department of Physics, Ohio State University, Columbus, OH 43210, USA \\ ${ }^{3}$ Department of Astronomy, Ohio State University, Columbus, \\ OH 43210, USA \\ ${ }^{4}$ Center for Cosmology and Astro-Particle Physics, Ohio State University, \\ Columbus, OH 43210, USA \\ ${ }^{5}$ Santa Cruz Institute for Particle Physics, University of California, \\ Santa Cruz, CA 95064, USA \\ ${ }^{6}$ Department of Physics and Astronomy, University of Rochester, \\ Rochester, NY 14627, USA \\ E-mail: ando@tapir.caltech.edu, beacom@mps.ohio-state.edu, \\ profumo@caltech.edu and rain@pas.rochester.edu
}

Received 20 November 2007

Accepted 26 March 2008

Published 21 April 2008

Online at stacks.iop.org/JCAP $/ 2008 / \mathrm{i}=04 / \mathrm{a}=029$

doi:10.1088/1475-7516/2008/04/029

\begin{abstract}
As suggested by some extensions of the standard model of particle physics, dark matter may be a super-weakly-interacting lightest stable particle, while the next-to-lightest particle (NLP) is charged and metastable. One could test such a possibility with neutrino telescopes, by detecting the charged NLPs produced in high-energy neutrino collisions with Earth matter. We study the production of charged NLPs by both atmospheric and astrophysical neutrinos; only the latter, which is largely uncertain and has not been detected yet, was the focus of previous studies. We compute the resulting fluxes of the charged NLPs, compare those of different origins and analyze the dependence on the underlying particle physics set-up. We point out that, even if the astrophysical neutrino flux is very small, atmospheric neutrinos, especially those from the prompt decay of charmed mesons, may provide a detectable flux of NLP pairs at neutrino telescopes such as IceCube. We also comment on the flux of charged
\end{abstract}


NLPs expected from proton-nucleon collisions and show that, for theoretically motivated and phenomenologically viable models, it is typically subdominant and below detectable rates.

Keywords: cosmic rays, dark matter, solar and atmospheric neutrinos, neutrino and gamma astronomy

ArXiv ePrint: 0711.2908

\section{Contents}

1. Introduction 2

2. High-energy neutrino flux 6

2.1. High-energy astrophysical neutrinos from extra-galactic sources . . . . . . 6

2.2. Atmospheric conventional neutrinos . . . . . . . . . . . . . . . 7

2.3. Atmospheric prompt-decay neutrinos . . . . . . . . . . . . . 7

$\begin{array}{ll}\text { 3. Interaction cross section } & 8\end{array}$

4. Flux of long-lived staus $\quad 12$

4.1. Formulation . . . . . . . . . . . . . . . . . . 13

4.2. Thick target approximation . . . . . . . . . . . . . 15

4.3. Results of numerical integration: stau spectrum . . . . . . . . . . . 17

4.4. Stau flux from nucleon-nucleon collisions . . . . . . . . . . . . . . . . . 19

5. Role of the supersymmetric particle spectrum 20

6. Conclusions 22

Acknowledgments $\quad 23$

References $\quad 23$

\section{Introduction}

The fundamental nature of dark matter poses a profound challenge to contemporary theoretical particle physics. Observations constrain the neutrino - the only electrically and color neutral non-baryonic elementary particle within the standard model of particle physics - to have a negligible contribution to the overall dark matter budget $[1,2]$. Dark matter is regarded as one of the most compelling hints towards new physics beyond the standard model. The question of its elementary essence has triggered enormous theoretical and phenomenological efforts [3].

The existence of suitable dark matter particle candidates in several theoretically cogent extensions of the standard model, like low-energy supersymmetry [4] or extradimensional scenarios $[5,6]$, focused a strong interest on a class of dark matter candidates known as weakly interacting massive particles (WIMPs). Similarly to other standard model particles, WIMPs would fall out of thermal equilibrium and freeze out in the early Universe, leaving a relic abundance compatible with the inferred amount of dark 
matter [7]. These WIMPs can be directly detected by experiments looking for the minuscule energy deposition caused by dark matter particles scattering nuclei [8]. The pair annihilation of WIMPs into energetic gamma rays, neutrinos and antimatter is a second, yet indirect, handle on the presence and potential imprint of galactic particle dark matter [9].

The connection of the aforementioned scenarios to the electroweak scale, soon to be probed at the Large Hadron Collider (LHC), motivated the exploration of complementarity between collider searches for new physics and the question of the elementary nature of dark matter [10]-[14]. In most cases, if dark matter is a WIMP, the anticipated experimental signature at the LHC would be the production of strongly interacting massive particles which promptly decay to the lightest and stable WIMP, plus a number of energetic jets and leptons. The neutral dark matter particle would escape the detector unobserved, leading to large missing transverse energy as well [15]. Conclusive identification of escaping neutral particles at the LHC with dark matter permeating our galaxy and other cosmic structures would, however, require some evidence from the direct and/or indirect WIMP searches listed before [9].

WIMPs are indeed attractive dark matter candidates, but are not the only theoretically envisioned possibility. The dark matter particle could exhibit even feebler interactions with ordinary standard model particles than a WIMP, making direct and indirect searches completely hopeless. For instance, the supersymmetric gravitino [16] or the Kaluza-Klein graviton of universal extra dimensions $[6,17]$ are perfectly plausible 'super-weakly-interacting' [18] dark matter candidates (super-WIMPs). If Nature chose an option like this, collider signatures of new physics, if any, would strongly depend upon the nature of the next-to-lightest supersymmetric particle (NLSP). Since a super-WIMP is also very weakly coupled to the other new physics heavier states, the NLSP would likely be quasi-stable. If the NLSP is neutral, the qualitative experimental landscape would look like that of a standard WIMP scenario. However, we would lack the needed proof of a connection between the weakly interacting long-lived particles produced at colliders and galactic dark matter.

If the NLSP is instead charged (constituting a charged massive particle, or CHAMP), the LHC would potentially observe the extremely distinct signature of a 'heavy muon': charged tracks and penetration of the outer muon subdetector, possibly at very low relativistic beta. CHAMPs are constrained by direct collider searches at LEP2 [19], as well as at the Tevatron $[20]^{7}$.

If a CHAMP were stable on collider scales, it could still decay on cosmological scales, and thus impact precision astrophysical measurements [22], including the chemical potential associated with the cosmic microwave background black-body spectrum [23], the extra-galactic gamma-ray background [24], the reionization history of the Universe [25], the formation of small scale structures $[26,27]$ and the synthesis of light elements in the early Universe $[28,29]$ (see, for implications of neutral particle, [30]). Anomalies in the above-mentioned quantities, however, could hardly be considered smoking gun evidence that the collider CHAMPs were indeed related to lighter, super-weakly-interacting dark matter.

\footnotetext{
7 These and future searches at the LHC are not trivial, but advanced work is being done to ensure that such potential signals would not be missed [21]. Detection for masses $\lesssim 1 \mathrm{TeV}$ is essentially guaranteed.
} 
If CHAMPs were stable on collider timescales, but featured a short lifetime on cosmological timescales, say of the order of a year or less, CHAMPs produced in colliders might be trapped in large water tanks surrounding the detectors [31]. The tanks would then be periodically drained to underground reservoirs where CHAMP decays might be observed in low-background conditions [31]. While certainly not straightforward experimentally, such a technique might provide important information first on the actual metastability of the charged species and, second, on the nature of the super-weaklyinteracting particle the CHAMP would decay into. However, even if CHAMP decays were actually observed, this would still not suffice as conclusive evidence that the elusive particle CHAMPs decay into is indeed the dark matter constituent.

To our knowledge, beyond high-energy collider experiments, the only direct experimental handle on a super-weakly-interacting dark matter particle featuring a heavier, metastable charged partner is CHAMP pair production via neutrino-nucleon collisions, followed by direct observation at neutrino telescopes. This idea, originally proposed in [32], relies on the fact that the energy losses of CHAMPs in the Earth are significantly smaller than those of muons, therefore CHAMP pairs (unlike muon pairs) can reach the detector even if they were produced far away. This makes the relevant target volume for neutrino-nucleon interactions much larger. The CHAMP pairs can be efficiently separated from muon pairs, due to large track separations in the detector. The original proposal was subsequently followed up by related studies [33]-[38], which focused on the specific case of a gravitino lightest supersymmetric particle (LSP), and a stau NLSP playing the role of the CHAMP. Among other aspects, these studies investigated in detail stau energy losses in the Earth and in the detector, computed expected event rates for a few sample models and the relevant background, and addressed the possibility of discriminating single-stau from single-muon events.

Given the steady progress in the deployment of next-generation $\mathrm{km}^{3}$-size neutrino telescopes - particularly IceCube at the South Pole, already under construction and taking data - we consider it timely to address in detail a few points relevant for improving our understanding of the prospects for implementing the above outlined technique. In particular, as background rejection is not a substantial issue, the crucial point appears to be the evaluation of the CHAMP pair event rate at IceCube. To this end, we focus on the following four aspects:

(i) Incoming neutrino flux. So far, all long-lived CHAMP analyses for neutrino telescopes have considered a relatively optimistic flux of astrophysical neutrinos - as large as the Waxman-Bahcall (WB) bound [39] — as the primary source for CHAMP pair production. However, the WB bound is only a theoretical upper limit on the flux of astrophysical neutrinos expected from optically thin sources; therefore, the absolute normalization as well as the spectral shape of the true astrophysical neutrino flux remain largely unknown (of course, the biggest reason for this is that these neutrinos are as yet undetected!). On the other hand, atmospheric neutrinos have been detected and their flux is rather accurately known (below $~ 100 \mathrm{TeV}[40]$ ). At larger energies $(\gtrsim 100 \mathrm{TeV})$, although there is no detection so far, one can rather reliably extrapolate the flux of conventional atmospheric neutrinos from lower energies. In addition, one also expects a significant flux of so-called prompt-decay atmospheric neutrinos, which originate from the decay of short-lived charmed mesons and feature a harder spectral index than the conventional component. While we again have only an upper limit 
on this prompt-decay component, we know from particle physics that it necessarily exists, at some level, in the high-energy regime, and will be measured accurately by IceCube. In any event, atmospheric conventional and prompt-decay neutrinos evidently contribute as well to CHAMP pair production in neutrino-nucleon collisions. In this paper, we study the role of these standard, guaranteed neutrino sources, and compare it to the contribution from astrophysical neutrino flux models including the WB bound.

(ii) Underlying particle physics model. The event rate depends not only on the incoming neutrino flux, but also on the nature of the assumed particle physics model. Here, we consider generic supersymmetric models featuring a gravitino LSP and stau NLSP, and study how the stau pair production cross section and event rates at neutrino telescopes depend on the given mass spectrum. Neutrino-nucleon interactions produce slepton-squark pair final states, the amplitudes mediated by supersymmetric fermion exchange (neutral and charged gauginos and higgsinos).

(iii) Other CHAMP sources. Unlike the high-energy neutrino flux, the flux of very energetic protons is accurately measured and well known up to extremely high energies; proton-nucleon interactions feature a supersymmetric pair production cross section significantly larger than that of neutrino-nucleon processes. It therefore seems reasonable to quantitatively assess the flux of stau pairs produced in the interaction of incident primary protons with nuclei in the atmosphere. The tradeoff is the enormous cross section for proton-nucleus scattering into standard model particles, which depletes the incoming proton flux, highly suppressing any stau pair production rate. Since quark-antiquark processes can directly produce stau pairs, however, the kinematic threshold for stau pair production, as a function of the incoming primary particle energy, is lower for proton-nucleus than for neutrinonucleus processes. Yet we find that, for reasonable and phenomenologically acceptable particle models, the expected stau pair event rates from proton-nucleus collisions are experimentally negligible at all energies, leaving neutrinos as the only relevant primary source particles for CHAMP pair production.

(iv) Simplified analytic approach. We present the computation of the flux of staus from neutrino-nucleon interactions from first principles and argue that, to an acceptable degree of accuracy, the total number of expected staus can be computed as one simple integral of three factors. Specifically, we show that the quantities of physical relevance are: (1) the incident flux of primary neutrinos, (2) the ratio of the cross sections of neutrino plus nucleon into supersymmetric particle pairs to the total neutrino-nucleon cross section and (3) a geometric efficiency factor.

Hereafter, we specifically use supersymmetric staus as charged metastable NLPs, but note that the following arguments are applicable to any other possible candidates of longlived CHAMPs. The outline of the remainder of this paper is as follows. We discuss the various components of the high-energy neutrino flux and their connected uncertainties in section 2. We introduce the new physics scenarios and compute the stau pair production cross sections in section 3. Section 4 outlines the computation of the stau event rate at IceCube, including the above-mentioned simplified analytic treatment. The stau rate dependence on the particle physics framework is addressed in section 5 and we draw conclusions in section 6 . 


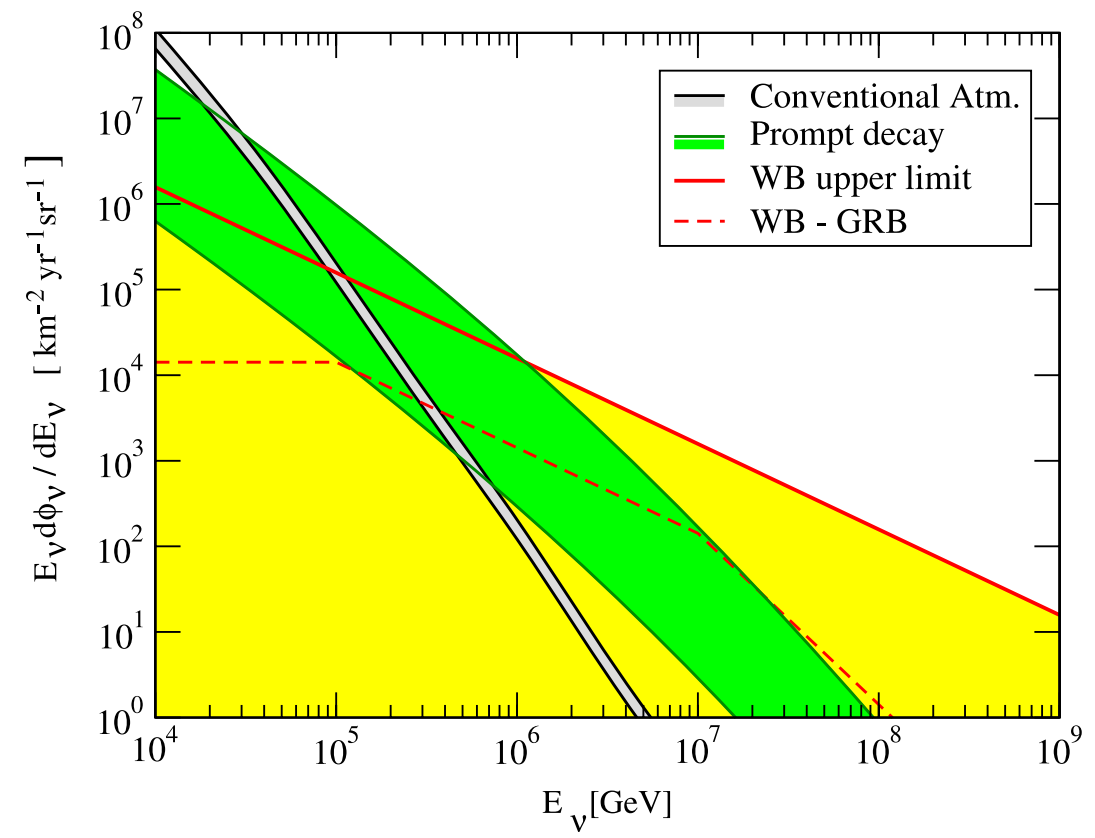

Figure 1. The differential flux of high-energy neutrinos (plus antineutrinos) considered in the present study, summed over neutrino flavors. The shaded gray, green and yellow regions indicate the uncertainty ranges for the fluxes of conventional atmospheric, prompt-decay atmospheric and astrophysical neutrinos, respectively. The reference extra-galactic neutrino fluxes refer to the WB limit [39] (red solid line) and to their prediction for GRBs [41] (red dashed line).

\section{High-energy neutrino flux}

In this section, we summarize the high-energy neutrino fluxes we consider in the present study. As discussed in section 1, past works considered only a flux of neutrinos close to, or saturating, the WB upper limit. However, other neutrino sources potentially contribute as well: these include conventional atmospheric neutrinos, prompt-decay atmospheric neutrinos and possibly astrophysical neutrinos other than those considered in the WB set-up. Since the stau production rate does not depend on neutrino flavor $^{8}$, all we care about is the total neutrino plus antineutrino flux, i.e. the flux of $\nu=\nu_{\mathrm{e}}+\nu_{\mu}+\nu_{\tau}$, where each $\nu_{\mathrm{i}}$ here indicates neutrino plus antineutrino of flavor $i$. From this point on, we mean this combined quantity when we use the term 'flux', unless otherwise stated. In figure 1, we summarize and collect the various neutrino sources we discuss below and the ranges of normalizations we consider.

\subsection{High-energy astrophysical neutrinos from extra-galactic sources}

Very powerful astrophysical objects such as active galactic nuclei (AGNs) and gamma-ray bursts (GRBs) are candidate high-energy neutrino sources. This is because strong gamma-

\footnotetext{
${ }^{8}$ Flavor is conserved in the underlying supersymmetric particle pair production event, but all supersymmetry particles then decay promptly to a stau plus standard model particles. Thus, an electron neutrino eventually produces a stau pair just as a tau neutrino does.
} 
ray emission detected from these objects can be attributed to the particle acceleration and successive interaction with the surrounding medium, magnetic and photon fields, which might also be a source of neutrinos via charged meson production.

If these neutrino sources are optically thin, then the upper bound on neutrino flux is obtained from the well-measured cosmic-ray flux, because each proton that arrives at the Earth should produce no more than a few neutrinos at the source. Based on this argument and assuming that the cosmic-ray spectrum above $10^{9} \mathrm{GeV}$ is of extragalactic origin, Waxman and Bahcall [39] derived the upper bound for the $\nu_{\mu}$ flux before neutrino oscillation to be $E_{\nu_{\mu}}^{2} \mathrm{~d} \Phi_{\nu_{\mu}}^{\mathrm{WB}} / \mathrm{d} E_{\nu_{\mu}}=(1-4) \times 10^{-8} \mathrm{GeV} \mathrm{cm}^{-2} \mathrm{~s}^{-1} \mathrm{sr}^{-1}$, where the range reflects cosmological evolution of source density. As we expect that flavor ratio at production (i.e. before oscillation) is $\nu_{\mathrm{e}}: \nu_{\mu}: \nu_{\tau}=1: 2: 0$ due to meson decays, the WB bound summed over flavors after neutrino oscillation is $E_{\nu}^{2} \mathrm{~d} \Phi_{\nu}^{\mathrm{WB}} / \mathrm{d} E_{\nu}=(1.5-6) \times$ $10^{-8} \mathrm{GeV} \mathrm{cm}^{-2} \mathrm{~s}^{-1} \mathrm{sr}^{-1}$. Here we adopt $E_{\nu}^{2} \mathrm{~d} \Phi_{\nu}^{\mathrm{WB}} / \mathrm{d} E_{\nu}=5 \times 10^{-8} \mathrm{GeV} \mathrm{cm}^{-2} \mathrm{~s}^{-1} \mathrm{sr}^{-1}$ for our reference value and show this bound in figure 1.

We stress that, although all previous studies adopted the WB upper limit on astrophysical neutrino flux as their incident source, that flux is not a model prediction, but an upper bound. One can never regard the output of a WB maximal neutrino flux as a solid prediction for the stau event rate at IceCube; the resulting stau flux is merely an upper limit. In addition, a few comments are in order on the WB bound's robustness, especially at our energies of interest. First, the WB bound is valid for $E_{\nu}>5 \times 10^{7} \mathrm{GeV}$, the threshold corresponding to proton energies of $10^{9} \mathrm{GeV}$ (a daughter neutrino carries $\sim 5 \%$ of its parent proton's energy [39]). Below this, the WB bound is only an extrapolation, since the cosmic-ray flux below $10^{9} \mathrm{GeV}$ is totally dominated by the galactic component. Second, by its definition the WB bound is applicable only to optically thin sources. If neutrino-emitting opaque objects existed, their contributions might sum to produce a neutrino flux exceeding the WB bound. Potential sources include baryon-rich GRBs [42] and starburst galaxies [43].

We also adopt a model for high-energy neutrino production via shocks in GRBs [41]. In contrast to the WB bound, it is a prediction; see the red dashed line in figure 1.

\subsection{Atmospheric conventional neutrinos}

While the astrophysical neutrino flux is totally unknown, there is a guaranteed and well-measured neutrino component - atmospheric neutrinos. These arise from the decays of mesons produced by cosmic rays striking the upper atmosphere. Neutrinos coming from pion and kaon decays form the 'conventional' component, which is well studied both theoretically [44] and experimentally [40]. Although there is no detection of any neutrinos for $E_{\nu} \gtrsim 10^{5} \mathrm{GeV}$, the energy range we are mainly interested in, this component should quite easily be extrapolated using measured data at lower energies, thus providing guaranteed seeds for CHAMP production. We use the model of [45] for the conventional atmospheric flux. As shown in figure 1, the well-known spectrum of these neutrinos falls rather steeply with increasing energy.

\subsection{Atmospheric prompt-decay neutrinos}

Hadronic cosmic-ray interactions with the Earth's atmosphere also produce short-lived charmed mesons [46]-[54]. Even though branching ratios into these final states are not 
large, the neutrino spectrum from the subsequent decays of charmed mesons is quite hard as they immediately decay, before losing energy. As a consequence, the contribution from this 'prompt-decay' component to the total flux of atmospheric neutrinos falls less rapidly with energy than the conventional component. The absolute normalization of this prompt flux is, however, still unknown, and there is a large range of model predictions. The main source of uncertainty is the proper treatment of next-to-leading-order charmed meson production cross sections, which strongly depend on the behavior of the nucleon parton distribution functions (PDFs). In our study, we consider a range between a smaller prompt flux from [52] and a larger prompt flux obtained by using the shape presented in [53] and normalizing it to IceCube's experimental upper limit [54]. Such a large flux, just allowed by the data, is in fact characteristic of the largest model predictions among [46]-[54]. We show these fluxes in figure 1.

\section{Interaction cross section}

Several TeV-scale extensions of the standard model feature a metastable massive charged particle. Perhaps the best-motivated scenario from a theoretical standpoint is supersymmetry, which provides several examples. If the LSP is very weakly interacting e.g. a gravitino or a right-handed sneutrino, where the interactions with the rest of the supersymmetric partners are suppressed by gravitational couplings or a gauge symmetry the NLSP is generally metastable. Specifically, a charged NLSP can occur in supergravity theories with a gravitino LSP $[16,55,56]$, gauge mediated supersymmetry breaking setups $[57,58]$, scenarios featuring a stau-neutralino near-degeneracy (particularly in the socalled co-annihilation region $[27,59]$; here one can have a neutralino LSP) or supergravity scenarios with a right-handed sneutrino LSP [60].

Another $\mathrm{TeV}$-scale new physics set-up that naturally encompasses a metastable NLP is that of universal extra dimensions (UED) with a Kaluza-Klein (KK) graviton as the lowest mass eigenstate in the KK tower [6]. In the minimal UED set-up, the next-tolightest $\mathrm{KK}$ state is usually neutral and corresponds to the KK first excitation of the $U(1)$ gauge boson, $B^{(1)}$, if the Higgs mass is below $\approx 200 \mathrm{GeV}$ for any value of the compactification inverse radius $R^{-1}$. However, as pointed out in [61], even in the minimal UED set-up the next-to-lightest KK particle (NLKP) can be charged and the LKP can be the $\mathrm{KK}$ graviton if $R^{-1} \lesssim 809 \mathrm{GeV}$ and $m_{\mathrm{h}} \gtrsim 250 \mathrm{GeV}$. In this case, the NLKP corresponds to the KK first charged Higgs mode. Alternatively, the boundary conditions at the orbifold fixed points can alter the spectrum and give rise to scenarios with a KK lepton as the NLKP, and again, a KK graviton LKP [6]. In general, electroweak precision observables constrain the scale $R^{-1}$ where the first excitation of a five-dimensional UED scenario might be expected to a few hundred $\mathrm{GeV}$, depending upon the value of the Higgs mass [62]. The analysis outlined below applies, with the appropriate production cross sections and energy losses, to such UED models and to any other similar framework featuring a metastable charged particle.

We choose to work with two of the well-known and well-motivated supersymmetric frameworks mentioned above: gauge mediated supersymmetry breaking (GMSB) $[57,58]$ and minimal supergravity (mSUGRA) with a gravitino LSP (see, e.g., $[16,55,56]$ ). For each framework we examine two models. One is a 'supersymmetric benchmark' (the SPS7 point of [63] for GMSB and the $\varepsilon$ model of [64] for mSUGRA with gravitino LSP) and the other is a variant with a lighter spectrum (models I and II). These are 
Table 1. The input parameters for the mSUGRA (upper pair) and GMSB (lower pair) models used in the present analysis and in figure 2 .

\begin{tabular}{llllll}
\hline mSUGRA models & $M_{1 / 2}(\mathrm{GeV})$ & $m_{0}(\mathrm{GeV})$ & $\tan \beta$ & $\operatorname{sgn}(\mu)$ & $A_{0}$ \\
\hline I & 280 & 10 & 11 & $>0$ & 0 \\
$\varepsilon[64]$ & 440 & 20 & 15 & $>0$ & $-25 \mathrm{GeV}$ \\
\hline GMSB models & $M_{\text {mes }}(\mathrm{TeV})$ & $\Lambda(\mathrm{TeV})$ & $\tan \beta$ & $\operatorname{sgn}(\mu)$ & $N_{\text {mes }}$ \\
\hline II & 70 & 35 & 15 & $>0$ & 3 \\
SPS7 [63] & 80 & 40 & 15 & $>0$ & 3 \\
\hline
\end{tabular}

Table 2. The masses of the lightest stau, first-generation squark, and lightest neutralino and chargino for the four models of table 1.

\begin{tabular}{lllll}
\hline Model & $m_{\widetilde{\tau}_{1}}(\mathrm{GeV})$ & $m_{\widetilde{q}_{1}}(\mathrm{GeV})$ & $m_{\widetilde{\chi}_{1}^{0}}(\mathrm{GeV})$ & $m_{\tilde{\chi}_{1}^{ \pm}}(\mathrm{GeV})$ \\
\hline $\mathbf{I}$ & 101 & 620 & 110 & 200 \\
$\boldsymbol{\varepsilon}[64]$ & 153 & 940 & 180 & 340 \\
II & 101 & 800 & 140 & 240 \\
SPS7 [63] & 120 & 900 & 160 & 270 \\
\hline
\end{tabular}

essentially rescalings of the first two. By adopting benchmark models, not only do we consider phenomenologically viable and theoretically soundly motivated set-ups, but we also make it easier to compare the detection technique discussed here with a wealth of existing phenomenological analyses of the same models (see, e.g., [63,64]). As will become apparent below, for the present analysis the details of the spectrum of the heavy supersymmetric particle pair produced is crucial. Assuming a degenerate sfermion spectrum, while potentially useful to get an understanding of the role of the mass scale in the expected size of the signal, can potentially be a misleading over-simplification.

We specify the mSUGRA and GMSB input parameters in table 1. Notice in particular that model II lies on the SPS7 slope defined in [63]. In table 2 we detail the four models' relevant particles masses.

The gravitino mass need not be specified as long as the stau decay length, $c \tau_{\widetilde{\tau}}$, is larger than or of the same order as the Earth's radius, $R_{\oplus}$. This implies a lower limit on the gravitino mass $m_{\widetilde{G}}[65]$ :

$$
c \tau_{\widetilde{\tau}} \simeq 1.7 \times 10^{9} \mathrm{~km}\left(\frac{m_{\widetilde{G}}}{100 \mathrm{GeV}}\right)^{2}\left(\frac{1 \mathrm{TeV}}{m_{\widetilde{\tau}}}\right)^{5}\left(1-\frac{m_{\widetilde{G}}^{2}}{m_{\widetilde{\tau}}^{2}}\right)^{-4} \gtrsim R_{\oplus} .
$$

Rearranged, the formula implies, for instance, that for a $100 \mathrm{GeV}$ stau the gravitino mass can be as light as $1 \mathrm{MeV}$, and for a $1 \mathrm{TeV}$ stau $m_{\widetilde{G}}$ has to be larger than $0.3 \mathrm{GeV}$. Notice that equation (1) does not take into account the relativistic boost factor, $\gamma$, which is sizable for staus produced in very high-energy neutrino-nucleon interactions. It is therefore a conservative constraint on the gravitino mass.

In addition, the stau lifetime should be short enough to be consistent with limits obtained from the effects on light element abundances processed in big-bang nucleosynthesis [66] (see also [67]) and from excessive distortions to the cosmic microwave 
background spectrum [68]. Of particular relevance are constraints resulting from overproduction of ${ }^{6} \mathrm{Li}$ and ${ }^{7} \mathrm{Li}[28,69,70]$, induced by catalytic effects produced by bound states consisting of light nuclei and the metastable CHAMP (here, the lightest stau). Notice, however, that the details of the estimate of the amount of primordially synthesized lithium are still under debate [71]. Also, the constraints depend upon the fraction of electromagnetic energy released in the decay. Conservatively, if one requires the lifetime of the charged metastable species to be shorter than $10^{3}-10^{4} \mathrm{~s}$, as implied by the analysis of [72], the gravitino mass is constrained to be approximately below $1 \mathrm{GeV}$ for a $100 \mathrm{GeV}$ stau and below $100 \mathrm{GeV}$ for a $1 \mathrm{TeV}$ stau. This evidently leaves a very wide window, of almost three orders of magnitude, for the viable gravitino mass range.

To calculate the stau flux ${ }^{9}$, we first need to calculate the stau production cross section as a function of incoming neutrino energy. We do this using the SUSY-MADEVENT package $[73,74]$, which calculates the differential or total cross section for any $2 \rightarrow n$ scattering process in the MSSM given an SLHA-conforming (standardized format for spectra) model input file [75]. We generate model input files using the sUSPECT spectrum generator package [76], which also automatically checks the generated model against various known precision data constraints, such as $b \rightarrow s \gamma$. Our four models do not conflict with any known constraints. The processes contributing to stau production mainly stem from tree-level $u$ and $t$ exchange diagrams with a slepton $(\tilde{l}$ and $\tilde{\nu})$ plus a squark $(\tilde{u}$ and $\tilde{d})$ in the final state, through neutralino $\left(\chi^{0}\right)$ or chargino $\left(\chi^{ \pm}\right)$exchange, as discussed in [33]. More specifically, they are: $\nu+u(d) \stackrel{\chi^{ \pm}}{\rightarrow} \tilde{l}+\tilde{d}(\tilde{u}), \nu+u(d) \stackrel{\chi^{0}}{\rightarrow} \tilde{\nu}+\tilde{u}(\tilde{d})$, where $\chi^{0}$ and $\chi^{ \pm}$ indicate neutralinos and charginos, respectively. The squarks and sleptons produced in the final state promptly cascade decay into metastable staus.

We calculate total cross sections, summing over neutrino and antineutrino inelastic scattering on protons using exact matrix elements to supersymmetric pair final states for both charged current (CC) and neutral current (NC), employing CTEQ6L1 PDFs [77]. The possible final states are (anti-)sneutrino or (anti-)slepton, plus (anti-)squark. Our calculations are leading order, as there are no available NLO QCD corrections for neutrino-proton $(\nu \mathrm{p})$ scattering. Judging from the known results for proton-proton $(\mathrm{pp})$ scattering, however, we probably make an underestimate of the rate of the order of $50 \%$. In this regard our calculation is conservative. For our purposes at the relevant energies, the neutrino-neutron cross section is sufficiently close to the neutrino-proton cross section that we can ignore calculating it separately.

An interesting side observation is that the $\nu \mathrm{p}$ and $\bar{\nu} \mathrm{p}$ cross sections are not equal except at very large $\sqrt{s}$, where low- $x$ quarks dominate the PDFs and are approximately egalitarian [78]; we reproduce this observation here. As $\sqrt{s}$ gets within a couple of orders of magnitude above threshold, however, $\bar{\nu}$ p dominates because the larger CC process picks out valence quarks, where $u$ dominates slightly over $d$. Closer to threshold this remains true for the $\mathrm{CC}$ process, but for $\mathrm{NC} \nu \mathrm{p}$ dominates because of chirality selection for the final state squarks. Near threshold these two diverging components accidentally roughly cancel, resulting in $\sigma_{\nu \mathrm{p}} \approx \sigma_{\bar{\nu} \mathrm{p}}$ once again. For the present purposes, however, we are concerned only with total rates and ignore charge-separated subsamples, which would vary somewhat as a function of stau energy.

\footnotetext{
${ }^{9}$ Here the word 'stau' denotes staus and antistaus collectively. This is because both could be produced by the same interaction, but cannot be distinguished at neutrino telescopes.
} 

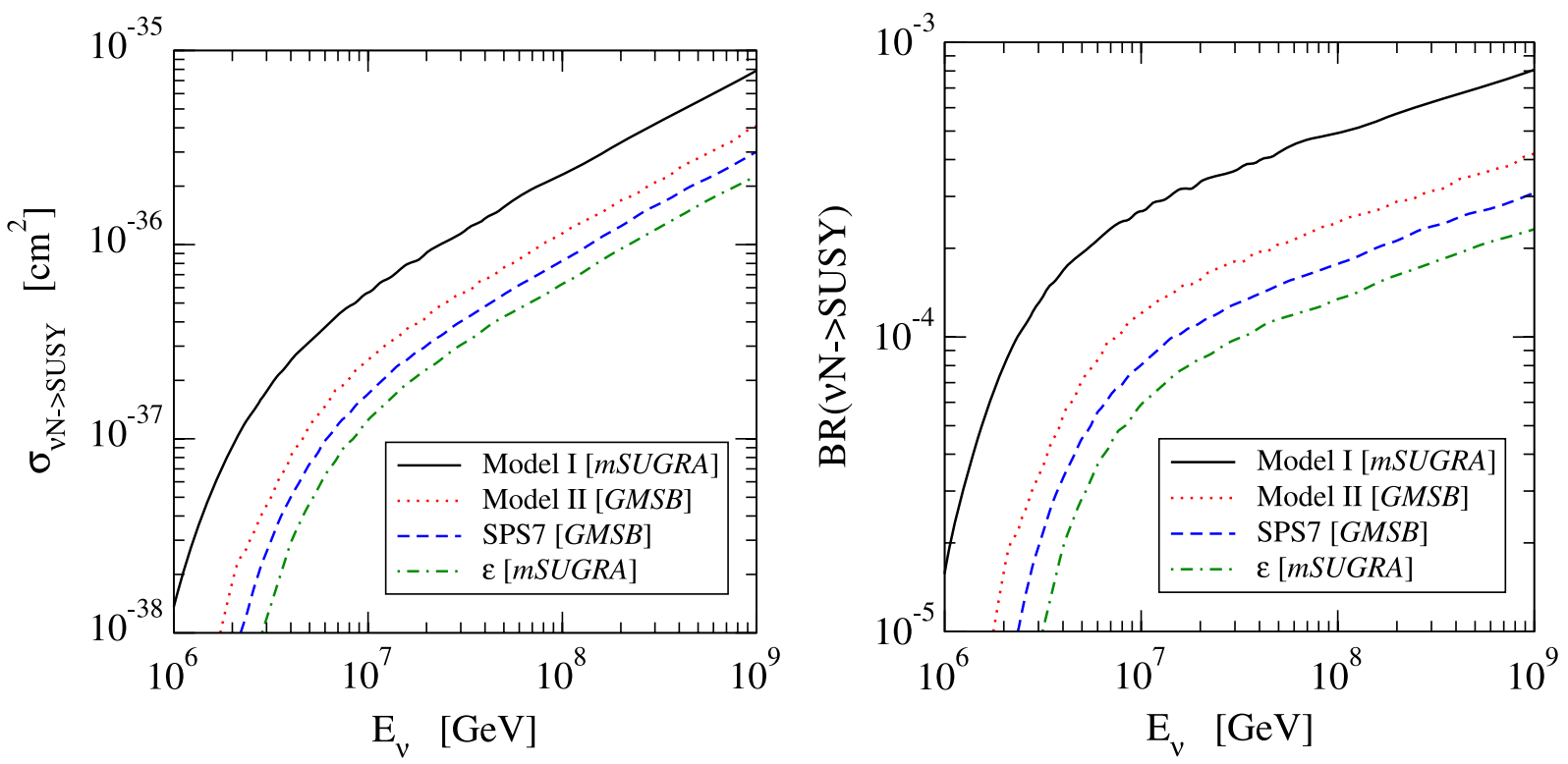

Figure 2. Left: the neutrino-proton cross sections, as a function of the incident neutrino energy for the production of stau pairs in the four models under consideration here (see the text and tables 1 and 2 for details on the specific models $)$. Right: the ratio $\mathrm{BR}(\nu \mathrm{N} \rightarrow \mathrm{SUSY})$ of the neutrino-proton cross section into supersymmetric particle pairs over the total neutrino-proton cross section, as a function of the incident neutrino energy, for the same four models as in the left panel.

The left panel of figure 2 illustrates our results for the neutrino-proton scattering cross section $\sigma_{\nu \mathrm{p}}\left(E_{\nu}\right)$ into any supersymmetric particle pairs, for the four models listed in table 1 , as a function of the incident neutrino energy. The general trends in $\sigma_{\nu \mathrm{p}}\left(E_{\nu}\right)$ are consistent with those found in other analyses, see, e.g., [33]: the steep rise in the low-energy end reflects the strong kinematic suppression associated with the squarkslepton pair production threshold. The subsequent rise of the cross section with the incoming neutrino energy depends upon the small- $x$ behavior of the PDFs. We give in section 5, where we discuss the role of the specific supersymmetric particle spectrum in the determination of $\sigma_{\nu \mathrm{p}}\left(E_{\nu}\right) \rightarrow \mathrm{SUSY}$, an analytical interpretation of the specific powerlaw behavior that emerges from the numerical computation. Notice that, compared to the optimistic toy model used in [33], theoretically motivated (optimistic) supersymmetric set-ups appear to give a maximal $\sigma_{\nu \mathrm{p}}$ that is roughly one order of magnitude smaller in the asymptotic high-energy regime.

In the right panel of figure 2 , we show the ratio of the neutrino-nucleon cross section into supersymmetric particle pairs over the total neutrino-nucleon cross section (that is, as apparent from the figure, always close to the purely standard model cross section). As we explain in section 4.2 , this is the physical quantity of interest, in the limit of the Earth as a thick target for high-energy neutrinos, for the computation of the stau flux. Beyond threshold effects, we point out that in the energy range of interest the branching ratio of neutrino-nucleon interactions into supersymmetric particle pairs lies between $10^{-4}$ and $10^{-3}$ 
Long-lived charged particles produced by atmospheric and astrophysical neutrinos
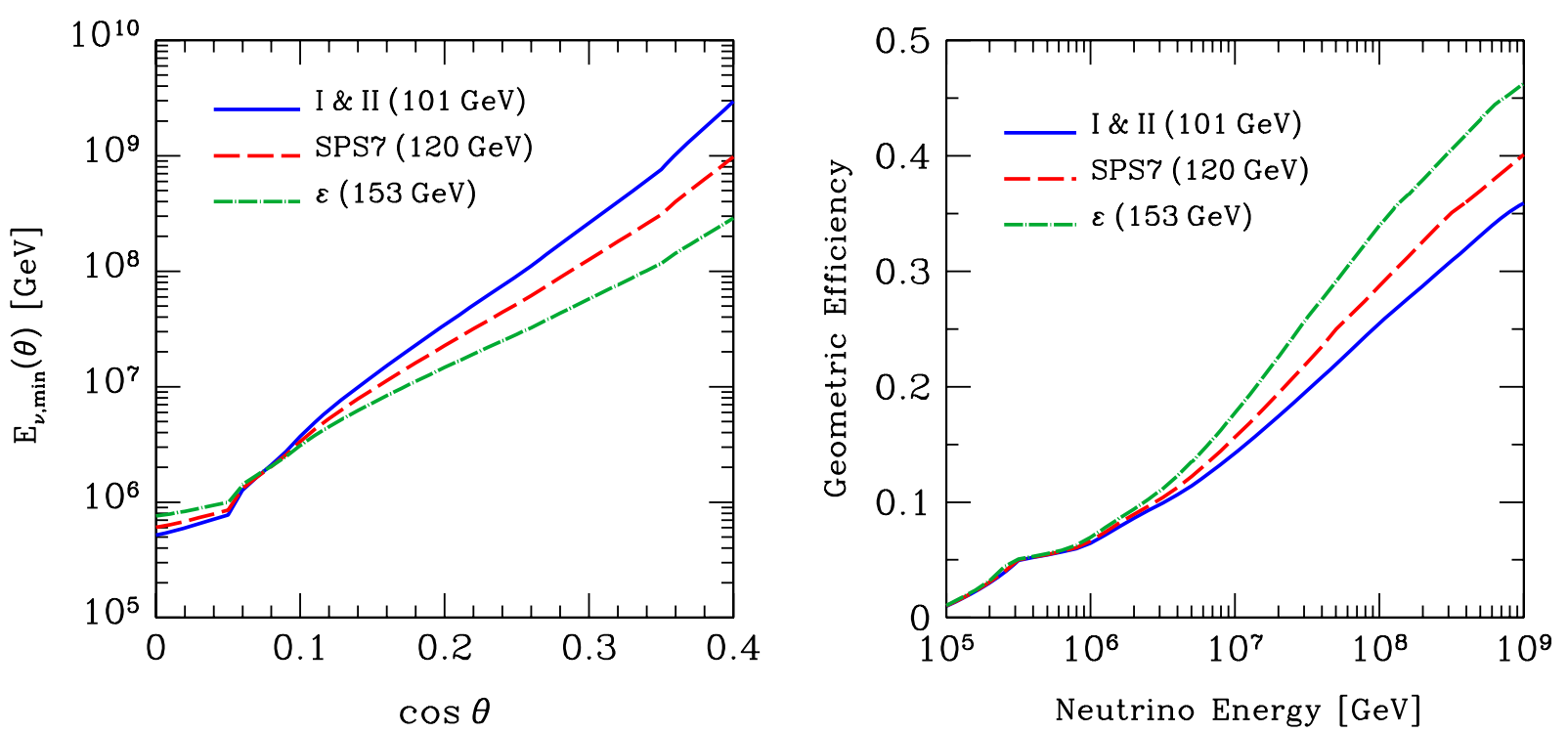

Figure 3. Left: minimum neutrino energy, $E_{\nu, \min }(\theta)$, below which the produced staus cannot reach the detector, as a function of the nadir angle $\theta$. Right: the geometric efficiency factor $\epsilon_{\text {geo }}\left(E_{\nu}\right)$ as a function of the incident neutrino energy (see equation (10)).

\section{Flux of long-lived staus}

We devote this section to a detailed analytical treatment of the computation of the flux of staus produced by neutrino-nucleon collisions that might be detected at a neutrino telescope such as IceCube. We start, in section 4.1, with a derivation of the differential flux of staus from first principles, leading to the result presented in equation (6). In the following section 4.2 we assume that the Earth is opaque to neutrinos at energies relevant here. In this thick target approximation, we analytically show that the flux of staus at the detector can be computed as a simple integral over incident neutrino energies, shown in equation (10), of the product of three factors:

(i) the differential flux of incident neutrinos (shown in figure 1),

(ii) the ratio of the neutrino-nucleon cross section into supersymmetric particles over the total neutrino-nucleon cross section (shown in figure 2, right) and

(iii) a 'geometric efficiency' factor, to be defined below and explicitly shown in the right panel of figure 3 .

When a neutrino interaction occurs, the branching ratio for stau production among the final states is given by the above cross section ratio. In the thick target approximation, all incoming neutrinos below the horizon will interact in the Earth. Since the staus are collinear with the incoming neutrino direction, and are produced with a sizable fraction of the neutrino energy, this leads to a simple but important result about the stau flux. Neglecting stau energy losses in matter for a moment, we see that the Earth acts as a neutrino-to-stau converter, with a probability that is independent of direction (below the horizon). This gives an upper bound on the stau flux through IceCube. The relevant range of energies will be between threshold $\left(\simeq 10^{6} \mathrm{GeV}\right.$; see figure 2$)$ and the point at which 
Table 3. Stau fluxes from various neutrino sources for the four benchmark supersymmetry models, in the thick target approximation, in units of $\mathrm{km}^{-2} \mathrm{yr}^{-1}$.

\begin{tabular}{lllll}
\hline Model & \multicolumn{1}{c}{ WB bound } & WB GRB & Atm. Prompt & Atm. Conv. \\
\hline I & $<3.2$ & 0.20 & $0.012-0.73$ & $0.0023-0.0038$ \\
II & $<1.2$ & 0.066 & $0.0028-0.16$ & $0.00028-0.00048$ \\
SPS7 & $<0.88$ & 0.045 & $0.0018-0.099$ & $0.00014-0.00024$ \\
$\boldsymbol{\varepsilon}$ & $<0.71$ & 0.034 & $0.0011-0.062$ & $0.000069-0.00012$ \\
\hline
\end{tabular}

Table 4. The stau flux from various neutrino sources for the four supersymmetry benchmark models, obtained from the exact numerical integration of equation (6), in units of $\mathrm{km}^{-2} \mathrm{yr}^{-1}$.

\begin{tabular}{lllll}
\hline Model & WB bound & WB GRB & Atm. Prompt & Atm. Conv. \\
\hline I & $<2.2$ & 0.13 & $0.0070-0.41$ & $0.0010-0.0018$ \\
II & $<0.93$ & 0.049 & $0.0019-0.11$ & $0.00017-0.00029$ \\
SPS7 & $<0.70$ & 0.035 & $0.0012-0.070$ & $0.000091-0.00015$ \\
$\varepsilon$ & $<0.57$ & 0.027 & $0.00080-0.047$ & $0.000049-0.000082$ \\
\hline
\end{tabular}

the stau flux becomes too small $\left(\simeq 10^{7} \mathrm{GeV}\right.$; note the product of figures 1,2$)$. Taking stau energy losses into account will only reduce the stau flux at the detector. Since staus cannot reach the detector from too far away, this means that only a limited range of nadir angles will be relevant, and this defines our geometric efficiency.

With our approximations, we give a preliminary assessment of the total expected stau flux at the detector (table 3). Finally, we compute the actual accurate stau flux resulting from the full-glory integration of equation (6) in section 4.3. We provide numerical estimates of the integrated flux in table 4 (that fall within a factor of two of the approximate results anticipated in table 3), as well as the actual differential flux of staus from the different primary incident neutrino sources. Finally, we comment in section 4.4 on the flux of staus predicted from nucleon-nucleon reactions.

\subsection{Formulation}

In the framework we consider here, all supersymmetric particles produced in neutrinonucleon interactions promptly decay into the NLSPs - here, the stau, which is metastable: long-lived enough to propagate through the Earth (with energy loss) and reach the detector. Our objective is to calculate the spectrum of staus after this energy loss, following similar principles for the spectra of neutrino-induced muons $[79,80]$.

The stau electromagnetic energy-loss rate is given by $[32,35]$

$$
\frac{\mathrm{d} E_{\mathrm{s}}}{\mathrm{d} X}=\alpha_{\mathrm{s}}+\beta_{\mathrm{s}} E_{\mathrm{s}}
$$

where $X=\rho \times l$ is the column depth of matter in units of $\mathrm{g} \mathrm{cm}^{-2}$ (i.e. density times distance), and the $\alpha_{\mathrm{s}}$ and $\beta_{\mathrm{s}}$ terms represent ionization and radiation losses, respectively. We neglect discrete scattering by weak interactions, as the effect is small at the energies we focus on near threshold [36]. Hereafter, we use the subscript $s$ to indicate quantities 
referring to staus. Our coordinate system locates the detector at $X=0$ and particles are produced at $X>0$, so that the energy $E_{\mathrm{s}}$ is a growing function of $X\left(\mathrm{~d} E_{\mathrm{s}} / \mathrm{d} X\right.$ is positive). For the density profile of the Earth, we use the model given in [81].

The ionization coefficient for staus, $\alpha_{\mathrm{s}}$, is approximately the same as that of muons [35]; specifically, we use $\alpha_{\mathrm{s}}=2 \times 10^{-3} \mathrm{GeV} \mathrm{cm}^{2} \mathrm{~g}^{-1}$. Radiative losses, on the other hand, depend on particle mass, and we take the corresponding coefficient to be given by $\beta_{\mathrm{s}}=4.2 \times 10^{-6}\left(m_{\mu} / m_{\mathrm{s}}\right) \mathrm{cm}^{2} \mathrm{~g}^{-1}$ [35]. By integrating equation (2), the 'distance' $X_{\mathrm{if}}=X_{\mathrm{i}}-X_{\mathrm{f}}$ traversed by the stau while its energy decreases from $E_{\mathrm{s}}^{\mathrm{i}}$ to $E_{\mathrm{s}}^{\mathrm{f}}$ is

$$
X_{\mathrm{if}}=\frac{1}{\beta_{\mathrm{s}}} \ln \left(\frac{\alpha_{\mathrm{s}}+\beta_{\mathrm{s}} E_{\mathrm{s}}^{\mathrm{i}}}{\alpha_{\mathrm{s}}+\beta_{\mathrm{s}} E_{\mathrm{s}}^{\mathrm{f}}}\right) .
$$

We assume that each stau produced in $\nu$ p interactions carries a large fraction of the parent neutrino energy. We denote this as $E_{\mathrm{s}}^{\mathrm{i}}=(1-y) E_{\nu}$ and assume $y=0.5$, independent of neutrino energy. (We discuss in section 4.2 the dependence of the stau flux on the parameter $y$.) At these high laboratory energies, the staus may be taken to be collinear with the original neutrino direction. The differential flux of produced staus per energy $E_{\mathrm{s}}^{\mathrm{i}}$ and distance $X$ is

$$
h_{\mathrm{s}}\left(E_{\mathrm{s}}^{\mathrm{i}}\right)=\frac{\mathrm{d}^{2} \Phi_{\mathrm{s}}^{\mathrm{i}}}{\mathrm{d} E_{\mathrm{s}}^{\mathrm{i}} \mathrm{d} X}=\frac{\sigma_{\mathrm{SUSY}}\left(E_{\nu}\right)}{(1-y) m_{p}} \frac{\mathrm{d} \Phi_{\nu}\left(E_{\nu}\right)}{\mathrm{d} E_{\nu}} \exp \left[-\frac{\left(X_{\max }-X\right) \sigma_{\mathrm{tot}}\left(E_{\nu}\right)}{m_{p}}\right],
$$

where $E_{\nu}=E_{\mathrm{s}}^{\mathrm{i}} /(1-y)$. The exponential factor takes into account the neutrino attenuation, mainly due to the standard model interactions $\left(\sigma_{\text {tot }} \approx \sigma_{\mathrm{SM}}\right.$; see figure 2 , right); $X_{\max }$ represents the value of $X$ corresponding to the surface of the Earth, which depends on the direction. The overall $(1-y)^{-1}$ factor comes from the change of variables from the spectrum of neutrinos to that of produced staus, i.e. $\mathrm{d} E_{\nu} / \mathrm{d} E_{\mathrm{s}}^{\mathrm{i}}=(1-y)^{-1}$.

The spectrum of staus at the detector is given by a double integral over all production positions and energies, subject to the constraint of having the stau energy be between $E_{\mathrm{s}}^{\mathrm{f}}$ and $E_{\mathrm{s}}^{\mathrm{f}}+\mathrm{d} E_{\mathrm{s}}^{\mathrm{f}}$ at $X=0$ :

$$
\frac{\mathrm{d} \Phi_{\mathrm{s}}^{\mathrm{f}}}{\mathrm{d} E_{\mathrm{s}}^{\mathrm{f}}}=\int_{E_{\mathrm{s}}^{\mathrm{f}}}^{\infty} \mathrm{d} E_{\mathrm{s}}^{\mathrm{i}} \int_{0}^{\infty} \mathrm{d} X h\left(E_{\mathrm{s}}^{\mathrm{i}}\right) \delta\left(E_{\mathrm{s}}^{\mathrm{f}}-f\left(E_{\mathrm{s}}^{\mathrm{i}}, X\right)\right),
$$

where the function $f\left(E_{\mathrm{s}}^{\mathrm{i}}, X\right)$ is defined by the energy loss, equation (3). We use the energy constraint to perform the $E_{\mathrm{s}}^{\mathrm{i}}$ integration, and as a result we obtain the following expression:

$$
\begin{aligned}
E_{\mathrm{s}}^{\mathrm{f}} \frac{\mathrm{d} \Phi_{\mathrm{s}}^{\mathrm{f}}}{\mathrm{d} E_{\mathrm{s}}^{\mathrm{f}}}=\int_{0}^{X_{\max }} & \mathrm{d} X\left[\frac{\exp \left[-\left(X_{\max }-X\right) \sigma_{\mathrm{SM}}\left(E_{\nu}\right) / m_{p}\right]}{m_{p} / \sigma_{\mathrm{SM}}\left(E_{\nu}\right)}\right] \\
\times & {\left[\left(\frac{E_{\mathrm{s}}^{\mathrm{f}}}{E_{\mathrm{s}}^{\mathrm{i}}}\right)\left(E_{\nu} \frac{\mathrm{d} \Phi_{\nu}}{\mathrm{d} E_{\nu}}\right)\left(\frac{\sigma_{\mathrm{SUSY}}\left(E_{\nu}\right)}{\sigma_{\mathrm{SM}}\left(E_{\nu}\right)}\right) \mathrm{e}^{\beta_{\mathrm{s}} X}\right], }
\end{aligned}
$$

where $E_{\nu}$ and $E_{\mathrm{s}}^{\mathrm{i}}$ inside the integral have to be evaluated according to the chosen $E_{\mathrm{s}}^{\mathrm{f}}$ on the left-hand side and the $X$ at that step inside the integral. From the energy-loss equation, equation (3), we have

$$
E_{\mathrm{s}}^{\mathrm{i}}=\left(E_{\mathrm{s}}^{\mathrm{f}}+\frac{\alpha_{\mathrm{s}}}{\beta_{\mathrm{s}}}\right) \mathrm{e}^{\beta_{\mathrm{s}} X}-\frac{\alpha_{\mathrm{s}}}{\beta_{\mathrm{s}}},
$$

where, again from the kinematic definition, $E_{\nu}=E_{\mathrm{s}}^{\mathrm{i}} /(1-y)$. 


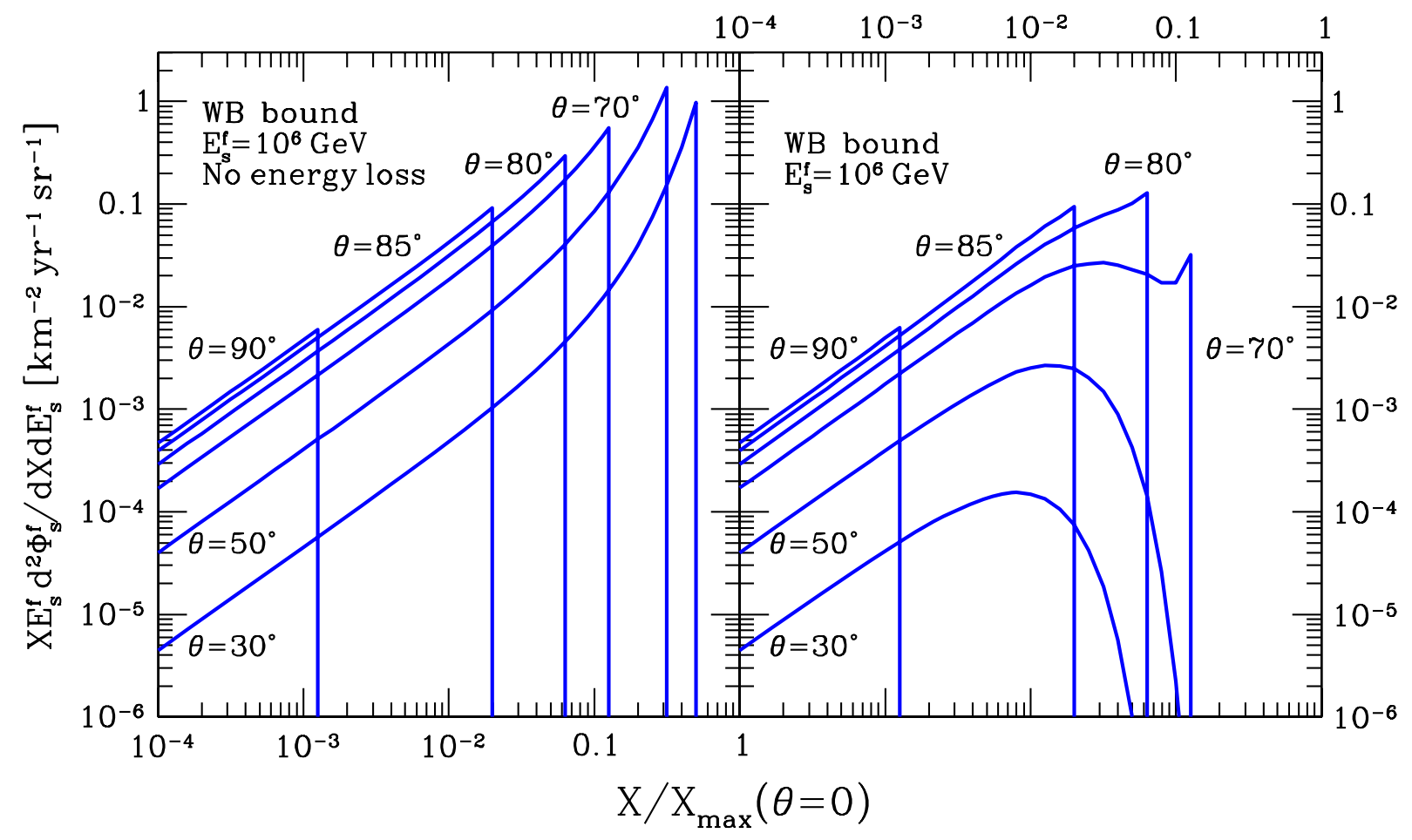

Figure 4. The contribution to the stau events from a given column depth $X$, at an energy $E_{\mathrm{s}}^{\mathrm{f}}=10^{6} \mathrm{GeV}$, for an incident neutrino flux saturating the WB bound. Each curve corresponds to a different value of the nadir angle $\theta$ as labeled. The left panel is shown with the assumption that there is no energy loss for staus, just for illustration purposes. In the right panel, proper energy losses are included.

It is convenient to change the integration variable by dividing the differential $\mathrm{d} X$ by $X$ and multiplying the integrand by $X$. Then the integration steps are in $\ln X$, and in figure 4 we show this new integrand for different nadir angles. In the left-hand panel, the stau energy losses in matter are neglected, so that the neutrino interaction and geometric effects are shown clearly. For each nadir angle $\left(90^{\circ}\right.$ is at the horizon and $0^{\circ}$ is along the diameter of the Earth), the sharp edges at large $X$ occur due to the boundary of the Earth. The peaks arise because the neutrino interactions occur logarithmically near $X=X_{\max }$. Substantial attenuation of the neutrino flux is only seen at small nadir angles; in units of the axis, the exponential scale height is $\simeq 0.1$. In the right-hand panel, the stau energy losses are now included. As expected, this prevents staus from arriving at the detector from too large distances (beyond $\simeq 0.01-0.05$ in units of the axis, depending on nadir angle due to the radial variation of the density profile). In this panel, the visual area under each curve shows its relative importance to the total stau flux through the detector.

\subsection{Thick target approximation}

For neutrino energies relevant for our purposes, $E_{\nu} \gtrsim 10^{6} \mathrm{GeV}$, the Earth is opaque to neutrinos at the most important nadir angles. Thus, to a good approximation, we can assume that staus are produced from neutrino interactions logarithmically near the 
Earth's surface. In this case, the exponential factor in equation (6) is sharply peaked at $X=X_{\max }$, i.e. $\exp \left[-\left(X_{\max }-X\right) \sigma_{\mathrm{SM}} / m_{p}\right] /\left(m_{p} / \sigma_{\mathrm{SM}}\right) \simeq \delta\left(X-X_{\max }\right)$. For our change of variables, the integrand is proportional to $\delta\left(\ln X-\ln X_{\max }\right)=X \delta\left(X-X_{\max }\right)$.

We then obtain

$$
E_{\mathrm{s}}^{\mathrm{f}} \frac{\mathrm{d} \Phi_{\mathrm{s}}^{\mathrm{f}}}{\mathrm{d} E_{\mathrm{s}}^{\mathrm{f}}} \simeq \frac{E_{\mathrm{s}}^{\mathrm{f}}}{E_{\mathrm{s}}^{\mathrm{i}}}\left(E_{\nu} \frac{\mathrm{d} \Phi_{\nu}}{\mathrm{d} E_{\nu}}\right) \frac{\sigma_{\mathrm{SUSY}}}{\sigma_{\mathrm{SM}}} \mathrm{e}^{\beta X_{\max }}=\frac{E_{\mathrm{s}}^{\mathrm{f}}}{E_{\mathrm{s}}^{\mathrm{i}}} \frac{\mathrm{d} E_{\mathrm{s}}^{\mathrm{i}}}{\mathrm{d} E_{\mathrm{s}}^{\mathrm{f}}}\left(E_{\nu} \frac{\mathrm{d} \Phi_{\nu}}{\mathrm{d} E_{\nu}}\right) \frac{\sigma_{\mathrm{SUSY}}}{\sigma_{\mathrm{SM}}},
$$

where, in the second equality, we used the relation $\mathrm{d} E_{\mathrm{s}}^{\mathrm{i}} / \mathrm{d} E_{\mathrm{s}}^{\mathrm{f}}=\mathrm{e}^{\beta X_{\max }}$ from equation (7) with $X=X_{\max }$.

We require the staus to be more energetic than a given detector threshold $E_{\mathrm{s}, \mathrm{th}}$. If the staus are relativistic enough to emit Cerenkov light, they will be detected. We adopt $E_{\mathrm{s}, \mathrm{th}}=300 \mathrm{GeV}$, since the typical stau mass we consider is $\sim 100 \mathrm{GeV}$, but we note that our results change negligibly for higher or lower thresholds. For instance, a shift by one order of magnitude, $E_{\mathrm{s}, \mathrm{h}}=3 \mathrm{TeV}$, affects the final result by only $0.2 \%$.

To satisfy the detection requirement $E_{\mathrm{s}}^{\mathrm{f}}>E_{\mathrm{s}, \mathrm{th}}$, the initial stau and corresponding neutrino energies must be larger than some minima, $E_{\mathrm{s}, \min }^{\mathrm{i}}$ and $E_{\nu, \min }$, given by

$$
E_{\mathrm{s}, \min }^{\mathrm{i}}(\theta)=\left(E_{\mathrm{s}, \mathrm{th}}+\frac{\alpha_{\mathrm{s}}}{\beta_{\mathrm{s}}}\right) \mathrm{e}^{\beta_{\mathrm{s}} X_{\max }(\theta)}-\frac{\alpha_{\mathrm{s}}}{\beta_{\mathrm{s}}}, \quad E_{\nu, \min }(\theta)=\frac{E_{\mathrm{s}, \min }^{\mathrm{i}}(\theta)}{1-y},
$$

as evident from equation (7). We plot this minimal neutrino energy as a function of the nadir angle $\theta$ in the left panel of figure 3 for the various supersymmetry models under consideration. Note that the crucial quantity here is the stau mass; hence we obtain the same result for models $\mathbf{I}$ and II. In the thick target approximation, the flux of staus reaching the detector from below is thus given by

$$
\begin{aligned}
F_{\mathrm{s}} & =\int_{\theta<\pi / 2} \mathrm{~d} \Omega \int_{E_{\mathrm{s}, \mathrm{th}}}^{\infty} \mathrm{d} E_{\mathrm{s}}^{\mathrm{f}} \frac{\mathrm{d} \Phi_{\mathrm{s}}^{\mathrm{f}}}{\mathrm{d} E_{\mathrm{s}}^{\mathrm{f}}} \\
& =\int_{\theta<\pi / 2} \mathrm{~d} \Omega \int_{0}^{\infty} \mathrm{d} E_{\nu}\left(\frac{\mathrm{d} \Phi_{\nu}}{\mathrm{d} E_{\nu}}\right)\left(\frac{\sigma_{\mathrm{SUSY}}}{\sigma_{\mathrm{SM}}}\right) \Theta\left(E_{\nu}-E_{\nu, \min }(\theta)\right) \\
& \equiv 2 \pi \int_{0}^{\infty} \mathrm{d} E_{\nu}\left(\frac{\mathrm{d} \Phi_{\nu}}{\mathrm{d} E_{\nu}}\right)\left(\frac{\sigma_{\mathrm{SUSY}}}{\sigma_{\mathrm{SM}}}\right) \epsilon_{\mathrm{geo}}\left(E_{\nu}\right),
\end{aligned}
$$

where $\Theta$ is the step function, and in the second equality we have simply used the definitions given above. In the last equality, we are defining a 'geometric efficiency' factor $\epsilon_{\text {geo }}\left(E_{\nu}\right)$, assuming that the incident neutrino intensity $\Phi_{\nu}$ is isotropic. We show this geometric efficiency factor in the right panel of figure 3. Thus, under the thick target approximation, the detection flux of staus can be divided into three independent factors:

- incident neutrino spectrum $\mathrm{d} \Phi_{\nu} / \mathrm{d} E_{\nu}$,

- cross-section ratio $\sigma_{\mathrm{SUSY}} / \sigma_{\mathrm{SM}}$ and

- geometric efficiency $\epsilon_{\text {geo }}$.

These three factors are illustrated in figures 1-3. After integrating over the neutrino energy in equation (10), we obtain approximate stau fluxes at the detector, summarized in table 3 for the ranges of incident neutrino fluxes given in section 2 .

So far we worked with the assumption that $y=0.5$, i.e. each stau carries half of the incident neutrino energy. However, especially when including the complex 
pattern of chain decay of squarks and sleptons into staus, a smaller fraction of the maximally available energy is expected to be carried by the staus, implying a larger value for $y$. The precise value for $y$ is model-dependent and its detailed evaluation is beyond the scope of the present analysis. We thus investigate the dependence of the stau flux on the $y$ parameter, for simplicity under the thick target approximation. Our calculations show that the flux of staus, with an incoming flux saturating the WB bound, and with our benchmark supersymmetric model $\mathbf{I}$, is 3.2, 2.7 and $1.5 \mathrm{~km}^{-2} \mathrm{yr}^{-1}$ for $y=0.5,0.7$ and 0.95 , respectively. This shows that the stau flux changes at most a factor of $\sim 2$ for a wide range of $y$, which is well within other model uncertainties. The weak dependence we find stems from the fact that a larger value of $y$ requires larger neutrino energies to produce staus with a certain energy. In turn, at larger energies the incident flux is smaller, but the cross-section ratio is larger. The cancellation of these two effects results in the mild dependence we find. The same argument also applies when we evaluate the flux more accurately in the next subsection, where we thus again use the assumption $y=0.5$, for simplicity.

\subsection{Results of numerical integration: stau spectrum}

We now solve equation (6) numerically to obtain a more precise spectrum and rate estimate of staus at the detector, as well as to check that the approximation made in the previous subsection is reasonable. Before giving the final flux estimates, we start by investigating the generic structure of the integrand of equation (6).

Figure 4 shows the integrand of equation (6) as a function of the column depth $X$ for various values of the nadir angle $\theta$, assuming $E_{\mathrm{s}}^{\mathrm{f}}=10^{6} \mathrm{GeV}$. For definiteness, we show here the result only for a neutrino injection model saturating the WB upper limit. From the right panel of this figure, one can see that the stau events are dominated by directions with large enough nadir angle, specifically with $\theta \gtrsim 70^{\circ}$, so that the staus can reach the detector. On the other hand, for very large nadir angles, the contributions to the total event rate are modest, because the Earth is not completely opaque in those directions, even at these high energies.

Figure 5 shows the differential stau fluxes as a function of final stau energy. First, we note that nearly all of the detectable staus are well above the threshold required to be relativistic, which is a stau energy comparable to the stau mass. Only relativistic charged particles produce the Čerenkov light that IceCube can measure.

The energy loss of relativistic particles may be dominated by ionization or radiation, depending on whether the $\alpha$ term or the $\beta$ term dominates in equation (2), respectively. This transition for muons occurs at an energy $\alpha / \beta \simeq 500 \mathrm{GeV}$. For staus, it occurs at an energy a factor $\simeq m_{\mathrm{s}} / m_{\mu}$ higher, i.e. at least $10^{6} \mathrm{GeV}$. The energy loss associated with Cerenkov radiation is always negligible; on the other hand, the Cerenkov radiation per unit length is the same for all relativistic particles. Thus, for particles at any energy in the relativistic ionization-dominated regime, all tracks will look the same in IceCube.

At higher energies, in the radiation-dominated regime, there is additional Cerenkov radiation arising from relativistic electrons and positrons created in hard radiative processes. In this regime, one can indeed tell the energy of the primary particle by the intensity of the total Cerenkov radiation. For most of the relevant final stau energies shown in figure 1 , the staus will at most be only slightly in the radiative regime, and so 


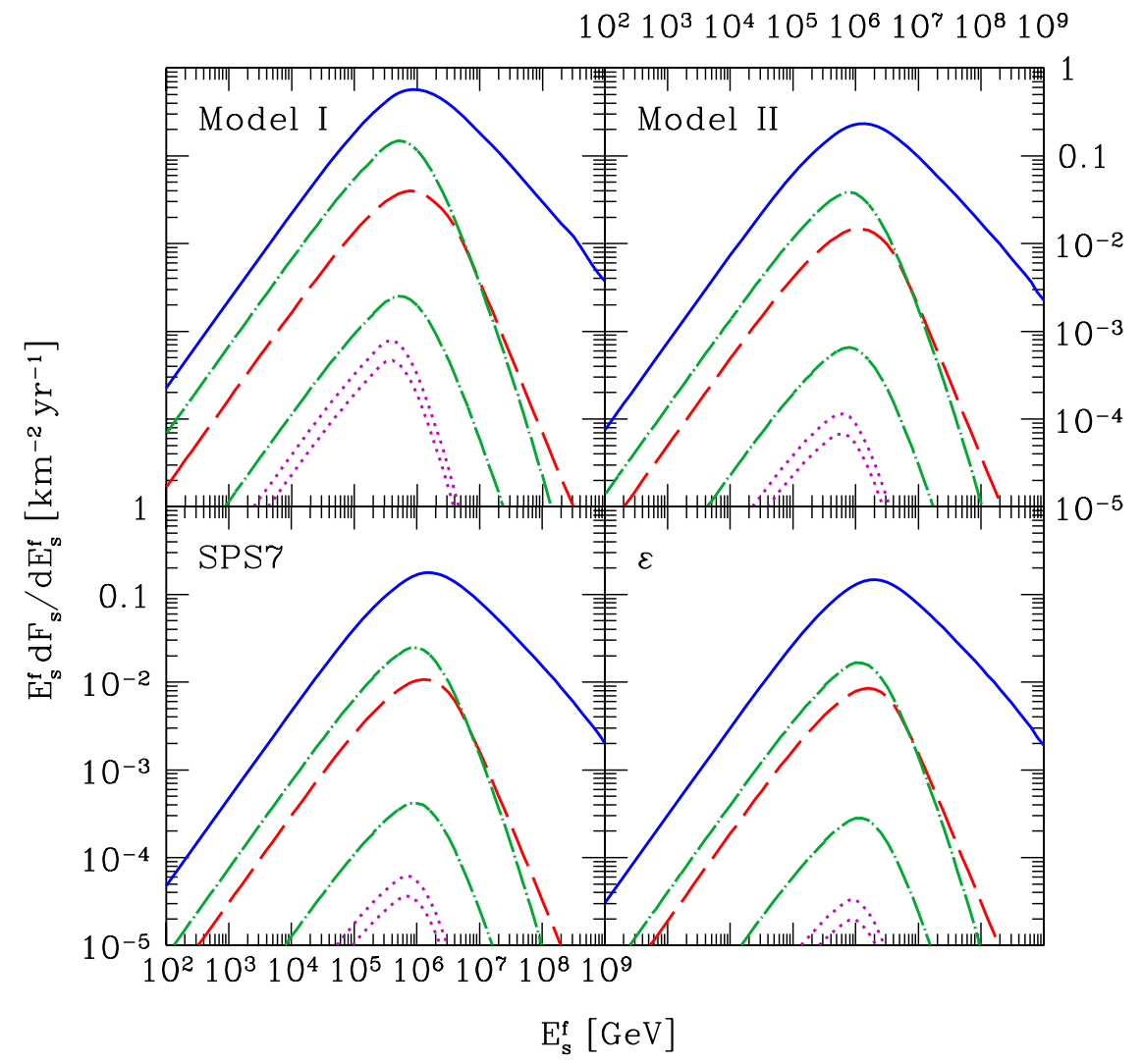

Figure 5. The differential stau flux at the detector for the four benchmark supersymmetry models. Each line corresponds to a different incident neutrino flux model: WB upper limit (blue solid), WB GRB (red dashed), atmospheric prompt (green dotted-dashed) and atmospheric conventional (magenta dotted).

all stau tracks going through IceCube will be indistinguishable from each other (and from low-energy muons). While the total energy deposited in the detector is much smaller for staus than it is for low-energy muons, this is irrelevant for IceCube, which detects only the Čerenkov light.

We argue that low-energy but relativistic stau pairs could also be detected (albeit without energy measurement), giving a sizable event rate. Recall that, while it might be difficult to distinguish between staus and muons on the basis of a single-particle detection, it would still be possible if we use dual-track events: since staus propagate over much longer distances in the Earth than muons, tracks entering the detector simultaneously are expected to be well separated [32]-[34]. The careful analysis of reference [34] shows that the separation distribution of stau pairs ranges widely, from $50 \mathrm{~m}$ to $1 \mathrm{~km}$, and that it peaks around $\sim 500 \mathrm{~m}$. On the other hand, the separation distribution for di-muons - the main background for the stau pair track-peaks at around $10 \mathrm{~m}$, and essentially no dimuon events with $>50 \mathrm{~m}$ separation are expected. Therefore, this criterion rejects almost all background di-muon events, but would capture a large fraction of stau events (typically $>50 \%$ ). Thus, we are interested in the stau flux integrated over energies larger than the relativistic threshold - which is very small, $E_{\mathrm{s}, \mathrm{th}} \simeq 300 \mathrm{GeV}$ - and where our results are least sensitive. This is clear from figure 5 . 
Table 4 shows the expected stau flux at the detector, obtained by integrating the spectrum above $300 \mathrm{GeV}$. Comparing with the results of table 3, where we used the thick target approximation, we find the two results are consistent with each other within a factor of 2, justifying the reliability of the thick target approximation.

Note that the flux of conventional atmospheric neutrinos is not totally isotropic, but peaks in the horizontal direction by almost one order of magnitude compared to other directions [45]. (The prompt flux is isotropic.) This is an important effect because most of the staus reaching the detector arose from neutrinos from horizontal directions (figure 4). Therefore, our results in tables 3 and 4 for atmospheric neutrinos would be larger by a factor of $\sim 3$, as these results were obtained with a direction-averaged incident neutrino flux.

As a consequence, given that the predicted stau flux could be as large as $\sim 1 \mathrm{~km}^{-2} \mathrm{yr}^{-1}$ and that one expects essentially no background from muon pair events, the search for stau pair tracks is warranted in the actual data. Our discussion here shows that a significant fraction of stau events could possibly come from atmospheric prompt-decay neutrinos (if the flux is close to the current upper bound), regardless of the assumed supersymmetry models.

\subsection{Stau flux from nucleon-nucleon collisions}

The stau pair flux $\Phi_{\mathrm{s}}$ produced from a differential flux of primary high-energy nucleons ${ }^{10}$ $\mathrm{d} \Phi_{\mathrm{N}} / \mathrm{d} E_{\mathrm{N}}$ colliding with atmospheric nuclei is given by

$$
\frac{\mathrm{d} \Phi_{\mathrm{s}}}{\mathrm{d} E_{\mathrm{s}}} \simeq \frac{\sigma_{\mathrm{NN}, \mathrm{SUSY}}}{\sigma_{\mathrm{NN}, \text { tot }}} \frac{\mathrm{d} \Phi_{\mathrm{N}}}{\mathrm{d} E_{\mathrm{N}}} \frac{\mathrm{d} E_{\mathrm{N}}}{\mathrm{d} E_{\mathrm{s}}}
$$

because the thick target approximation (introduced in section 4.2) is very good for $\mathrm{NN}$ interaction. The symbols $\sigma_{\mathrm{NN}, \text { tot }}$ and $\sigma_{\mathrm{NN}, \mathrm{SUSY}}$ indicate the total nucleon-nucleon interaction cross section and the cross section into any supersymmetric particle pair, respectively. To reiterate, since direct decays into gravitinos are strongly suppressed by gravitational couplings, all final state $R$ parity odd particles decay into the NLSP, i.e. lightest stau pairs. For the total NN cross section, we assume the parameterization for the hadron-air total cross section [82]:

$$
A \frac{\sigma_{\mathrm{NN}, \mathrm{tot}}}{\mathrm{mb}} \approx 185+13.3 \log \left(\frac{E_{\mathrm{N}}}{\mathrm{GeV}}\right)+0.08 \log ^{2}\left(\frac{E_{\mathrm{N}}}{\mathrm{GeV}}\right),
$$

where $A \simeq 14.6$ is the average number of nucleons in a nucleus of air. We approximate the nucleon-nucleon cross section into supersymmetric particles with the proton-proton cross section, and we compute the latter using Prospino2.0 [83]. For the incoming nucleon flux we use the estimate in figure 1 of [82].

Quark-antiquark processes can produce stau pairs directly, unlike neutrino-quark processes, where the final state has a larger threshold as the final state must contain a typically heavier squark. Hence the kinematic threshold, as a function of the primary particle energy, is lower in nucleon-nucleon collisions than in neutrino-nucleon collisions.

\footnotetext{
${ }^{10}$ Secondary nucleons and other hadrons contribute a small fraction of the incoming flux, and taking them into
} account does not affect our conclusions. 
Also, the subsequent occurrence of various supersymmetric particle thresholds at larger and larger masses, including particles featuring large degeneracy factors (such as squarks), implies a more rapidly growing behavior for the $\sigma_{\mathrm{NN}, \mathrm{SUSY}}$ cross section than that for $\sigma_{\nu \mathrm{N}, \mathrm{SUSY}}$. As a consequence, we find that, for the models under consideration here, $E_{\mathrm{p}} \mathrm{d} N_{\mathrm{s}} / \mathrm{d} E_{\mathrm{p}}$ is almost constant over several orders of magnitude in $E_{\mathrm{p}}$.

The final flux of staus is, however, dramatically suppressed by the ratio $\sigma_{\mathrm{NN}, \mathrm{SUSY}} / \sigma_{\mathrm{NN}, \text { tot }}[84]$, even taking into account multiplicity effects in stau pair production or proton re-interactions. In particular, for the models we consider here, where the strongly interacting supersymmetric particles are typically much more massive than the NLSP, the combination of threshold effects and of the rapidly decreasing flux of incident primary protons leads to dramatically less optimistic predictions than those recently reported in [37]. There, the authors considered squarks and gluinos with extremely low masses (150 and $300 \mathrm{GeV}$ ), while the theoretically motivated benchmark models we use here feature squark and gluino masses between $600 \mathrm{GeV}$ and $1 \mathrm{TeV}$. While we agree with the numerical results reported in [37] when making the same assumptions on squark and gluino masses, we obtain much lower figures for the benchmark models we adopt here. Namely, we find that, for the two most optimistic models, I and II, we predict a stau flux in IceCube from nucleon-nucleon interactions of $10^{-4}$ and $3 \times 10^{-6} \mathrm{~km}^{-2} \mathrm{yr}^{-1}$, respectively-much lower than even the contribution from conventional atmospheric neutrinos. We believe that this relative smallness compared with that from the atmospheric incident neutrinos would be a rather model-independent feature.

\section{Role of the supersymmetric particle spectrum}

In the previous sections we focused on specific supersymmetric models. We now wish to address the model-independent question of how the stau pair rate at neutrino telescopes depends upon the supersymmetric particle masses. As we already pointed out, the stau pair flux depends upon the $\nu \mathrm{N} \rightarrow$ SUSY cross section: the relevant masses entering the cross section are those of the heavy pair produced, and those of the supersymmetric partners of the electroweak gauge bosons which mediate the charged- and neutral-current interactions responsible for slepton-squark production. In addition, the number of produced stau pairs as a function of incoming neutrino energy crucially depends on the final state kinematic threshold: the larger the latter, the smaller the flux of incoming neutrinos that can lead to stau pair production, hence a smaller expected stau pair rate.

To explore quantitatively the statements above, we employ a convenient phenomenological parameterization of the supersymmetric set-up at the low-energy scale, and no longer rely on a specific supersymmetry breaking framework. The left panel of figure 6 shows the stau production cross section variation in high-energy neutrinoproton collisions in the plane defined by the slepton ( $x$ axis) and squark ( $y$ axis) masses. For definiteness, we fix the relevant gaugino (respectively, bino and wino) masses to $m_{1}=1 \mathrm{TeV}$ and $m_{2}=2 \mathrm{TeV}$. We choose $\mu=1 \mathrm{TeV}, m_{A}=500 \mathrm{GeV}, \tan \beta=10$, set all trilinear scalar couplings to zero and further assume that the soft supersymmetry breaking scalar masses of sleptons and (separately) of squarks are degenerate, CP-conserving and flavor-diagonal. In the figure, we show iso-level curves at fixed values of the production cross section for staus at an incident neutrino energy of $10^{8} \mathrm{GeV}$, in order to avoid production threshold effects. As the figure illustrates, cross-section variation is very mild 

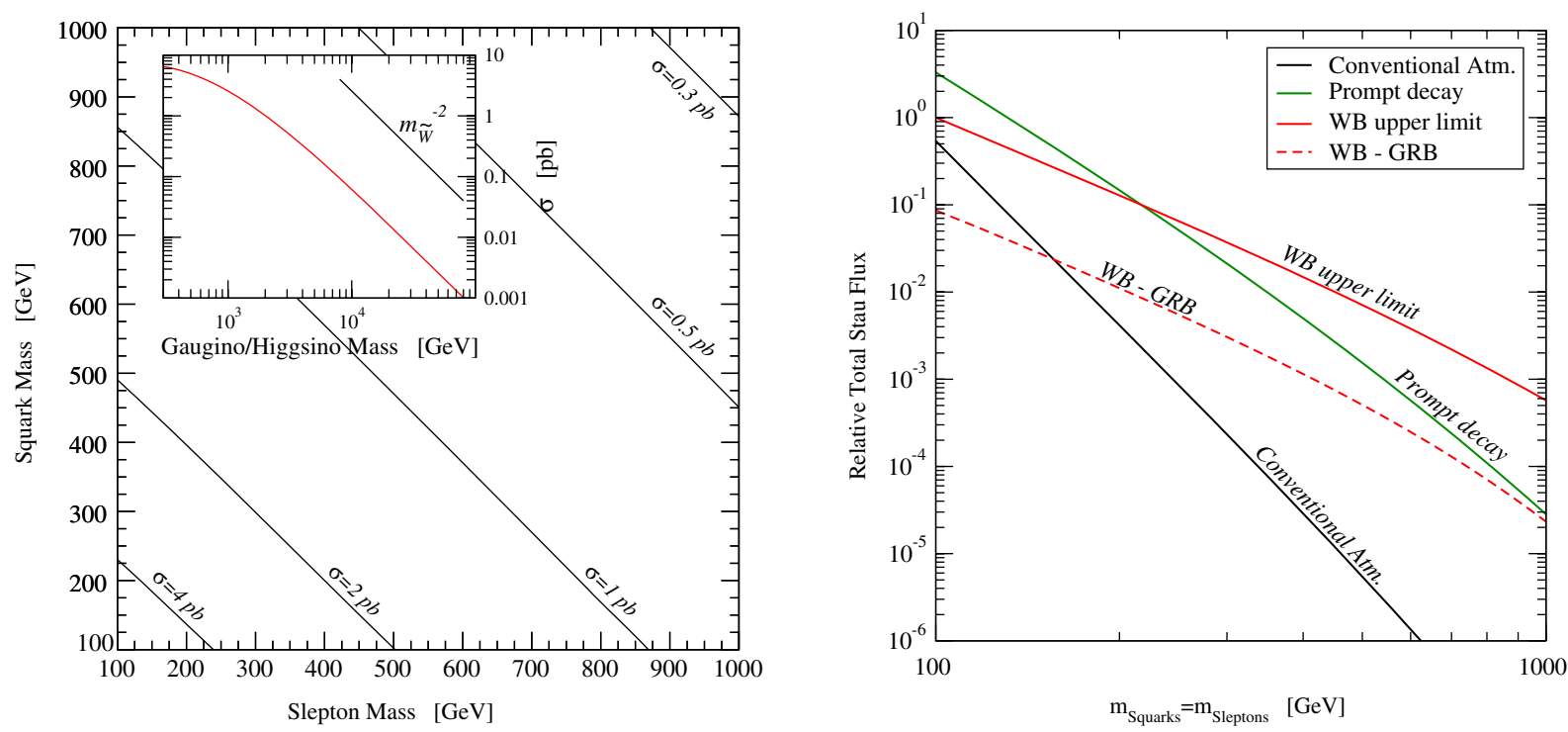

Figure 6. Left: isovalue curves (labeled) of the neutrino-proton cross section for supersymmetric particle pair production at incident $E_{\nu}=10^{8} \mathrm{GeV}$, in the plane defined by the slepton and squark masses (for simplicity we assume degenerate sleptons and degenerate squarks; see the text for details of the supersymmetric models). The inset illustrates the behavior of the cross section, again at $E_{\nu}=10^{8} \mathrm{GeV}$, as a function of a common gaugino/higgsino mass $\left(m_{1}=m_{2}=\mu\right)$. Right: contributions from conventional, prompt-decay and extra-galactic high-energy neutrinos to the total stau flux, as a function of the supersymmetric scalars' (degenerate) masses), normalized to be relative.

well above threshold. Quantitatively, the effect varies within little more than one order of magnitude for scalar masses varied between 100 and $1000 \mathrm{GeV}$.

In the inset, we show how the cross section scales the masses of $t$-channel supersymmetric particles (neutralinos and charginos) exchanged in squark-slepton pair production from neutrino-proton collisions. Neutralino and chargino masses are entirely determined at tree level by the gaugino soft supersymmetry breaking masses $m_{1}$ and $m_{2}$ and the higgsino mass term $\mu$, and by $\tan \beta$. For simplicity we assume a common 'gaugino/higgsino' mass scale, $m_{\widetilde{W}}$, defined as the common value of $m_{1}=m_{2}=\mu$. We employ a common scalar mass $m_{\mathrm{S}}$ for both sleptons and squarks of $300 \mathrm{GeV}$, and set all other low-scale supersymmetric parameters as in the rest of the figure $\left(m_{A}=500 \mathrm{GeV}\right.$, $\tan \beta=10$, all trilinear scalar couplings zero). As we illustrate in the inset, beyond the scalar mass scale $\left(m_{\widetilde{W}} \lesssim m_{\mathrm{S}}\right)$ where kinematic effects play a nontrivial role, cross-section scaling goes like the gaugino/higgsino mass scale to the power -2 . This can be analytically understood, since

$$
\begin{aligned}
\sigma_{\nu \mathrm{p}} & \sim \int_{4 m_{\mathrm{S}}^{2} / s}^{1} \mathrm{~d} x \int_{0}^{x s} \mathrm{~d} Q^{2} \frac{\mathrm{d}^{2} \sigma_{\nu \mathrm{p}}}{\mathrm{d} x \mathrm{~d} Q^{2}} \sim \int_{4 m_{\mathrm{S}}^{2} / s}^{1} \mathrm{~d} x \int_{0}^{x s} \mathrm{~d} Q^{2} \frac{\left[x \cdot q\left(x, Q^{2}\right)\right]}{\left(Q^{2}+m_{\widetilde{W}}^{2}\right)^{2}} \\
& =\int_{4 m_{\mathrm{S}}^{2} / s}^{1} \frac{x^{-1 / 3} s \mathrm{~d} x}{\left(m_{\widetilde{W}}^{4}+x s m_{\widetilde{W}}^{2}\right)^{2}},
\end{aligned}
$$


where, in equation (13), we made use of the fact that, in the large $E_{\nu}$ regime, $\left[x \cdot q\left(x, Q^{2}\right)\right] \sim$ $x^{-1 / 3}[85]$. One thus gets

$$
\sigma_{\nu \mathrm{p}} \sim \frac{1}{m_{\widetilde{W}}^{2}}\left(\frac{s}{4 m_{\mathrm{S}}^{2}}\right)^{1 / 3},
$$

which explains both the scaling in the inset of figure 6 and of $\sigma_{\nu \mathrm{p}}$ in figure 2 .

As the supersymmetric particle spectrum gets heavier, not only does the neutrinoproton cross section become smaller (left panel of figure 6), but more importantly the shift in incident neutrino energy threshold for stau production strongly suppresses the final stau flux. This depends on the dramatic dependence of the flux of incident neutrinos on energy, as illustrated in figure 1. The right panel of figure 6 quantifies this trend. There we show the relative contribution to the total stau flux from various incident neutrino fluxes as a function of the common squark and slepton masses $m_{\mathrm{S}}$. The fluxes are normalized to that resulting from the incident WB upper limit of extra-galactic neutrino flux and $m_{\mathrm{S}}=100 \mathrm{GeV}$. We set all the supersymmetric parameters to the same values as in the left panel. Comparing the relative flux for various origins summarized in table 4 with that shown in the right panel of figure 6 , we can roughly estimate that the four benchmark models correspond to $m_{\mathrm{S}} \approx 500 \mathrm{GeV}$ in the context of this phenomenological approach. In addition, recalling that the benchmark models predict a stau flux of $\sim 1 \mathrm{~km}^{-2} \mathrm{yr}^{-1}$ for the WB neutrino bound, this suggests that one can expect a stau flux well above $1 \mathrm{~km}^{-2} \mathrm{yr}^{-1}$, provided slepton and squark masses are smaller than $500 \mathrm{GeV}$.

The different neutrino flux scaling with energy dictates that the relative importance of the various neutrino sources depends on the mass scale of the particles produced in the neutrino-proton collision. In particular, while with a very light spectrum the contribution from conventional atmospheric neutrinos can be comparable to (or even larger than) the extra-galactic neutrino component, for $m_{\mathrm{S}} \gtrsim 0.5 \mathrm{TeV}$ the contribution from atmospheric neutrinos becomes negligible. Note that in that mass range the supersymmetric particles are so heavy that the overall stau flux is extremely suppressed, and likely undetectable. On the other hand, the figure illustrates that, in principle, prompt-decay neutrinos can be the dominant source of staus for almost any value of $m_{\mathrm{S}}$ if the extra-galactic neutrino flux is close to the GRB-derived range (dashed red line in the figure) rather than the WB upper limit. In addition, even conventional neutrinos contribute a stau flux of the same order of magnitude as that expected from the WB upper limit on astrophysical neutrinos, as long as the supersymmetric scalars mass scale is below $200 \mathrm{GeV}$. If both the prompt-decay neutrino flux and the extra-galactic neutrino flux are maximal, prompt neutrinos contribute at the same level as extra-galactic neutrinos for $m_{\mathrm{S}} \lesssim 400 \mathrm{GeV}$ and dominate for $m_{\mathrm{S}}$ below $200 \mathrm{GeV}$. Since a detectable signal is expected only for a light supersymmetric spectrum, this leads us to the following prediction: if the signal discussed here is indeed detected, a very sizable fraction of it will originate from conventional and prompt-decay neutrinos.

\section{Conclusions}

We reassessed the flux of metastable staus produced by neutrino-nucleon and nucleonnucleon interactions that might be detectable at $\mathrm{km}^{3}$ neutrino telescopes. We derived the flux of staus from first principles and showed that, under the approximation that the 
Earth is opaque to very high-energy neutrinos, the number of staus at the detector is given by a simple integral over the neutrino energy of the product of three factors: the incident neutrino flux, the ratio of the neutrino-nucleon cross section into supersymmetric particle pairs over the total neutrino-nucleon cross section and a geometric efficiency factor. We showed that this approximation reproduces an exact numerical computation within a factor of 2, which in turn is much better than the level of our knowledge of the first two factors entering the stau flux computation - namely, the incident neutrino flux and the features of the supersymmetric particle set-up.

We focused on each of the factors relevant to the computation of the final flux of staus. We concentrated on four well-motivated supersymmetric benchmark models, and independently evaluated the relevant production cross sections. We pointed out that previously neglected atmospheric neutrinos from prompt charmed meson decays could give a potentially large stau flux, even in the absence of a (yet to be discovered) astrophysical high-energy neutrino flux. This will depend on the prompt atmospheric neutrino flux being at the upper end of the theoretically expected range. Nucleon-nucleon processes, even for the most optimistic benchmark models, would not contribute sizably to the final stau flux. Finally, we numerically and analytically studied how the relevant cross sections depend on the supersymmetric model mass spectrum and how the relative importance of primary neutrino sources depends on the mass scale of supersymmetric scalars. In particular, we predict that, if the signal discussed here is indeed detected, a very sizable fraction of it would originate from conventional and prompt-decay neutrinos.

\section{Acknowledgments}

This work was supported by the Sherman Fairchild Foundation (SA); CCAPP, The Ohio State University, and NSF CAREER grant PHY-0547102 (JFB); DoE grants DE-FG0392-ER40701, DE-FG02-05ER41361, and NASA grant NNG05GF69G (SP); and DoE grant DE-FG02-91ER40685 (DR). We would like to thank Francis Halzen, Chris Quigg, and Xerxes Tata for enlightening discussions and Markus Ahlers for useful correspondence.

\section{References}

[1] Fukugita M, Ichikawa K, Kawasaki M and Lahav O, 2006 Phys. Rev. D 74027302 [SPIRES]

[2] Spergel D N et al (WMAP Collaboration), 2007 Astrophys. J. Suppl. 170377

[3] Bergstrom L, 2000 Rep. Prog. Phys. 63793 Bertone G, Hooper D and Silk J, 2005 Phys. Rep. 405279 [SPIRES]

[4] Jungman G, Kamionkowski M and Griest K, 1996 Phys. Rep. 267195 [SPIRES]

[5] Cheng H C, Feng J L and Matchev K T, 2002 Phys. Rev. Lett. 89211301 [SPIRES]

[6] Hooper D and Profumo S, 2007 Phys. Rep. 45329 [SPIRES]

[7] Wolfram S, 1979 Phys. Lett. B 8265 [SPIRES]

[8] Munoz C, 2004 Int. J. Mod. Phys. A 193093 [SPIRES]

[9] Bergstrom L, 1998 New Astron. Rev. 42245

[10] Drees M, Kim Y G, Nojiri M M, Toya D, Hasuko K and Kobayashi T, 2001 Phys. Rev. D 63035008 [SPIRES]

[11] Baer H, Balazs C, Belyaev A, Krupovnickas T and Tata X, 2003 J. High Energy Phys. JHEP06(2003)054 [SPIRES]

[12] Weiglein G et al (LHC/LC Study Group), 2006 Phys. Rep. 42647 [SPIRES]

[13] Masiero A, Profumo S and Ullio P, 2005 Nucl. Phys. B 71286 [SPIRES]

[14] Baltz E A, Battaglia M, Peskin M E and Wizansky T, 2006 Phys. Rev. D 74103521 [SPIRES]

[15] Baer H and Tata X, 2006 Weak Scale Supersymmetry: From Superfields to Scattering Events (Cambridge: Cambridge University Press)

[16] Blumenthal G R, Pagels H and Primack J R, 1982 Nature 29937 [SPIRES] 
[17] Feng J L, Rajaraman A and Takayama F, 2003 Phys. Rev. D 68085018 [SPIRES]

[18] Feng J L, Rajaraman A and Takayama F, 2003 Phys. Rev. Lett. 91011302 [SPIRES]

[19] Cerutti F et al (LEP2 SUSY Working Group), 2002 LEPSUSYWG/02-05.1 http://lepsusy.web.cern.ch/lepsusy/www/stable_summer02/stable_208.html

[20] Acosta D et al (CDF Collaboration), 2003 Phys. Rev. Lett. 90131801 [SPIRES]

[21] Fairbairn M et al, 2007 Phys. Rep. 4381 [SPIRES]

[22] See e.g. Chen X L and Kamionkowski M, 2004 Phys. Rev. D 70043502 [SPIRES]

[23] Zhang L, Chen X, Kamionkowski M, Si Z g and Zheng Z, 2007 Phys. Rev. D 76061301 [SPIRES]

[24] Cembranos J A R, Feng J L and Strigari L E, 2007 Preprint 0704.1658 [astro-ph]

[25] Pierpaoli E, 2004 Phys. Rev. Lett. 92031301 [SPIRES]

[26] Sigurdson K and Kamionkowski M, 2004 Phys. Rev. Lett. 92171302 [SPIRES]

[27] Profumo S, Sigurdson K, Ullio P and Kamionkowski M, 2005 Phys. Rev. D 71023518 [SPIRES]

[28] See, e.g. Kohri K and Takayama F, 2007 Phys. Rev. D 76063507 [SPIRES] Kawasaki M, Kohri K and Moroi T, 2007 Phys. Lett. B 649436 [SPIRES] Jittoh T, Kohri K, Koike M, Sato J, Shimomura T and Yamanaka M, 2007 Preprint 0704.2914 [hep-ph]

[29] Jedamzik K, 2007 Preprint 0707.2070 [astro-ph]

[30] Cumberbatch D, Ichikawa K, Kawasaki M, Kohri K, Silk J and Starkman G D, 2007 Preprint 0708.0095 [astro-ph]; and references therein

[31] Feng J L and Smith B T, 2005 Phys. Rev. D 71015004 Feng J L and Smith B T, 2005 Phys. Rev. D 71019904 (erratum)

[32] Albuquerque I, Burdman G and Chacko Z, 2004 Phys. Rev. Lett. 92221802 [SPIRES]

[33] Ahlers M, Kersten J and Ringwald A, 2006 J. Cosmol. Astropart. Phys. JCAP07(2006)005 [SPIRES]

[34] Albuquerque I F M, Burdman G and Chacko Z, 2007 Phys. Rev. D 75035006 [SPIRES]

[35] Reno M H, Sarcevic I and Su S, 2005 Astropart. Phys. 24107 [SPIRES]

[36] Huang Y, Reno M H, Sarcevic I and Uscinski J, 2006 Phys. Rev. D 74115009 [SPIRES]

[37] Ahlers M, Illana J I, Masip M and Meloni D, 2007 J. Cosmol. Astropart. Phys. JCAP08(2007)008 [SPIRES]

[38] Reno M H, Sarcevic I and Uscinski J, 2007 Preprint 0710.4954 [hep-ph]

[39] Waxman E and Bahcall J N, 1999 Phys. Rev. D 59023002 [SPIRES]

[40] Daum K et al (Frejus Collaboration), 1995 Z. Phys. C 66417 Ahrens J et al (AMANDA Collaboration), 2002 Phys. Rev. D 66012005 [SPIRES] Ashie Y et al (Super-Kamiokande Collaboration), 2005 Phys. Rev. D 71112005 [SPIRES]

[41] Waxman E and Bahcall J N, 1997 Phys. Rev. Lett. 782292 [SPIRES]

[42] Ando S and Beacom J F, 2005 Phys. Rev. Lett. 95061103 [SPIRES]

[43] Loeb A and Waxman E, 2006 J. Cosmol. Astropart. Phys. JCAP05(2006)003 [SPIRES] Stecker F W, 2007 Astropart. Phys. 26398 [SPIRES] Thompson T A, Quataert E, Waxman E and Loeb A, 2006 Preprint astro-ph/0608699

[44] Barr G D, Gaisser T K, Lipari P, Robbins S and Stanev T, 2004 Phys. Rev. D 70023006 [SPIRES] Barr G D, Gaisser T K, Robbins S and Stanev T, 2006 Phys. Rev. D 74094009 [SPIRES] Honda M, Kajita T, Kasahara K and Midorikawa S, 2004 Phys. Rev. D 70043008 [SPIRES] Honda M, Kajita T, Kasahara K, Midorikawa S and Sanuki T, 2007 Phys. Rev. D 75043006 [SPIRES]

[45] Candia J and Roulet E, 2003 J. Cosmol. Astropart. Phys. JCAP09(2003)005 [SPIRES]

[46] Zas E, Halzen F and Vazquez R A, 1993 Astropart. Phys. 1297 [SPIRES]

[47] Pasquali L, Reno M H and Sarcevic I, 1999 Phys. Rev. D 59034020 [SPIRES]

[48] Gelmini G, Gondolo P and Varieschi G, 2000 Phys. Rev. D 61056011 [SPIRES]

[49] Costa C G S, 2001 Astropart. Phys. 16193 [SPIRES]

[50] Volkova L V and Zatsepin G T, 2001 Yad. Fiz. 64313 [SPIRES] Volkova L V and Zatsepin G T, 2001 Phys. Atom. Nucl. 64266 [SPIRES] (translation)

[51] Fiorentini G, Naumov V A and Villante F L, 2001 Phys. Lett. B 510173 [SPIRES]

[52] Beacom J F and Candia J, 2004 J. Cosmol. Astropart. Phys. JCAP11(2004)009 [SPIRES]

[53] Martin A D, Ryskin M G and Stasto A M, 2003 Acta Phys. Pol. B 343273

[54] Achterberg A (IceCube Collaboration), 2006 Preprint astro-ph/0611597

[55] Feng J L, Su S and Takayama F, 2004 Phys. Rev. D 70075019 [SPIRES]

[56] Ellis J R, Olive K A, Santoso Y and Spanos V C, 2004 Phys. Lett. B 5887 [SPIRES] Ellis J R, Raklev A R and Oye O K, 2006 J. High Energy Phys. JHEP10(2006)061 [SPIRES] Ibarra A and Roy S, 2007 J. High Energy Phys. JHEP05(2007)059 [SPIRES]

[57] Dicus D A, Dutta B and Nandi S, 1997 Phys. Rev. D 565748 [SPIRES] Cheung K, Dicus D A, Dutta B and Nandi S, 1998 Phys. Rev. D 58015008 [SPIRES] 
Feng J L and Moroi T, 1998 Phys. Rev. D 58035001 [SPIRES]

Mercadante P G, Mizukoshi J K and Yamamoto H, 2001 Phys. Rev. D 64015005 [SPIRES]

[58] Giudice G F and Rattazzi R, 1999 Phys. Rep. 322419 [SPIRES]

[59] Ambrosanio S and Mele B, 1997 Phys. Rev. D 551399

Ambrosanio S and Mele B, 1997 Phys. Rev. D 563157 (erratum)

Gladyshev A V, Kazakov D I and Paucar M G, 2005 Mod. Phys. Lett. A 203085 [SPIRES]

Jittoh T, Sato J, Shimomura T and Yamanaka M, 2006 Phys. Rev. D 73055009 [SPIRES]

[60] Asaka T, Ishiwata K and Moroi T, 2006 Phys. Rev. D 73051301 [SPIRES]

Gupta S K, Mukhopadhyaya B and Rai S K, 2007 Phys. Rev. D 75075007 [SPIRES]

[61] Cembranos J A R, Feng J L and Strigari L E, 2007 Phys. Rev. D 75036004 [SPIRES]

[62] Flacke T, Hooper D and March-Russell J, 2006 Phys. Rev. D 73095002

Flacke T, Hooper D and March-Russell J, 2006 Phys. Rev. D 74019902 (erratum)

Gogoladze I and Macesanu C, 2006 Phys. Rev. D 74093012 [SPIRES]

[63] Allanach B C et al, 2001 Proc. APS/DPF/DPB Summer Study on the Future of Particle Physics (Snowmass, CO, Jun-Jul 2001) ed N Graf, p 125 [hep-ph/0202233]

[64] De Roeck A, Ellis J R, Gianotti F, Moortgat F, Olive K A and Pape L, 2007 Eur. Phys. J. C 491041 [hep-ph/0508198]

[65] Ellis J R, Olive K A, Santoso Y and Spanos V C, 2004 Phys. Lett. B 5887 [SPIRES]

[66] Cyburt R H, Ellis J R, Fields B D and Olive K A, 2003 Phys. Rev. D 67103521 [SPIRES] Jedamzik K, 2000 Phys. Rev. Lett. 843248 [SPIRES]

Feng J L, Su S f and Takayama F, 2004 Phys. Rev. D 70063514 [SPIRES]

Steffen F D, 2006 J. Cosmol. Astropart. Phys. JCAP09(2006)001 [SPIRES]

[67] Kawasaki M, Kohri K and Moroi T, 2005 Phys. Rev. D 71083502 [SPIRES]

Kawasaki M, Kohri K and Moroi T, 2005 Phys. Lett. B 6257 [SPIRES]

[68] Hu W and Silk J, 1993 Phys. Rev. Lett. 702661 [SPIRES]

[69] Pospelov M, 2007 Phys. Rev. Lett. 98231301 [SPIRES]

[70] Kaplinghat M and Rajaraman A, 2006 Phys. Rev. D 74103004 [SPIRES]

[71] Jedamzik K, 2007 Preprint 0707.2070 [astro-ph]

[72] Cyburt R H, Ellis J R, Fields B D, Olive K A and Spanos V C, 2006 J. Cosmol. Astropart. Phys. JCAP11(2006)014 [SPIRES]

[73] Cho G C, Hagiwara K, Kanzaki J, Plehn T, Rainwater D and Stelzer T, 2006 Phys. Rev. D 73054002 [SPIRES]

[74] Maltoni F and Stelzer T, 2003 J. High Energy Phys. JHEP02(2003)027 [SPIRES]

[75] Skands P et al, 2004 J. High Energy Phys. JHEP07(2004)036 [SPIRES]

Hahn T, 2004 Preprint hep-ph/0408283

Aguilar-Saavedra J A et al, 2006 Eur. Phys. J. C 4643

[76] Djouadi A, Kneur J L and Moultaka G, 2002 Preprint hep-ph/0211331

[77] Pumplin J, Stump D R, Huston J, Lai H L, Nadolsky P and Tung W K, 2002 J. High Energy Phys. JHEP07(2002)012 [SPIRES]

[78] Carena M, Choudhury D, Lola S and Quigg C, 1998 Phys. Rev. D 58095003 [SPIRES]

[79] Gaisser T K and Stanev T, 1985 Phys. Rev. D 312770 [SPIRES]

[80] Kistler M D and Beacom J F, 2006 Phys. Rev. D 74063007 [SPIRES]

[81] Gandhi R, Quigg C, Reno M H and Sarcevic I, 1996 Astropart. Phys. 581 [SPIRES]

[82] Illana J I, Masip M and Meloni D, 2007 Phys. Rev. D 75055002 [SPIRES]

[83] Beenakker W, Hopker R and Spira M, 1996 Preprint hep-ph/9611232

[84] Byrne M, Kolda C F and Regan P, 2002 Phys. Rev. D 66075007 [SPIRES]

[85] Gandhi R, Quigg C, Reno M H and Sarcevic I, 1998 Phys. Rev. D 58093009 [SPIRES] 